\title{
Parallel image restoration using surrogate constraint methods ${ }^{2}$
}

\author{
Bora Uçar ${ }^{\mathrm{a}}$, Cevdet Aykanat ${ }^{\mathrm{a}, *}$, Mustafa Ç. Pınar ${ }^{\mathrm{b}}$, Tahir Malas ${ }^{\mathrm{c}}$ \\ ${ }^{a}$ Department of Computer Engineering, Bilkent University, 06800 Ankara, Turkey \\ ${ }^{\mathrm{b}}$ Department of Industrial Engineering, Bilkent University, 06800 Ankara, Turkey \\ ${ }^{\mathrm{c}}$ Department of Electrical and Electronics Engineering, Bilkent University, 06800 Ankara, Turkey
}

Received 23 September 2005; received in revised form 5 July 2006; accepted 12 October 2006

\begin{abstract}
When formulated as a system of linear inequalities, the image restoration problem yields huge, unstructured, sparse matrices even for images of small size. To solve the image restoration problem, we use the surrogate constraint methods that can work efficiently for large problems. Among variants of the surrogate constraint method, we consider a basic method performing a single block projection in each step and a coarse-grain parallel version making simultaneous block projections. Using several state-of-the-art partitioning strategies and adopting different communication models, we develop competing parallel implementations of the two methods. The implementations are evaluated based on the per iteration performance and on the overall performance. The experimental results on a PC cluster reveal that the proposed parallelization schemes are quite beneficial.
\end{abstract}

(C) 2006 Elsevier Inc. All rights reserved.

Keywords: Parallel computing; Image restoration; Linear feasibility problem; Surrogate constraint method

\section{Introduction}

The purpose of the present paper is to use state-of-the-art parallel algorithms for the restoration of heavily distorted digital images. An ideal recording device would be expected to record an image with the following idealized property: the intensity of a pixel of the recorded image should be directly proportional to the corresponding section of the scene being recorded. However, this property is rarely observed in practice. Either the recorded intensity of a pixel is related to the intensity in a larger neighborhood of the corresponding section of the scene (blurring), or the recorded intensities are contaminated by random noise $[21,27]$. Image restoration is concerned with estimating the original scene from a distorted and noisy one. Restoration of images that have been blurred by various factors is usually posed as a linear estimation problem obtained from a discretization process, where the characteristics of the blurring system and the noise are assumed to be known a

\footnotetext{
This work is partially supported by the Scientific and Technological Research Council of Turkey (TÜBITAK) under project EEEAG-106E069.

* Corresponding author. Fax: +903122664047.

E-mail addresses: ubora@cs.bilkent.edu.tr (B. Uçar), aykanat@cs.bilkent.edu.tr (C. Aykanat),mustafap@ie.bilkent.edu.tr (M.Ç. Pınar), tahir@bilkent.edu.tr (T. Malas).
}

priori [21]. The mathematical model of the recording operation which is usually an integral equation is discretized to yield a linear system that is solved by a host of direct and iterative methods $[12,22,27,28]$. Among these methods, we focus on iterative methods. The advantage of iterative methods is that they allow a flexible and improved formulation of the restoration problem [21], and the large dimensions involved in image restoration make these methods favorable.

We follow the work [30] and pose the image restoration problem as a linear feasibility problem [8]. The linear feasibility problem asks for a point that satisfies a set of linear inequalities. In matrix notation, given an $M \times N$ matrix $A$ and $M \times 1$ vector $b$, the problem is to find an $N \times 1$ vector $x$ such that

$$
A x \leqslant b .
$$

We use iterative methods for solving the linear feasibility problem. An important class of iterative methods for the linear feasibility problem is the projection methods developed for the solution of the linear systems by Kacmarz (see [32]), and Cimmino (see [8]). Kacmarz's and Cimmino's works are extended to linear inequalities by Gubin et al. [11] and Censor and Elfving [7]. The method in [11] is known as the successive orthogonal projections method. In this method, an initial guess is successively projected onto hyperplanes corresponding to the 
boundary of the violated constraints until a feasible point satisfying all inequalities is found. The method in [7] is known as the simultaneous orthogonal projections method, where the current point is projected onto each of the violated constraint's hyperplanes simultaneously, and the new point is taken to be the convex combination of all projections.

Kacmarz and Cimmino type methods become computationally very expensive when applied to image restoration problem, mainly because a projection is made for each violated constraint. The surrogate constraint methods proposed by Yang and Murty [35] and the one that we use here eliminate this problem by processing a group of violated constraints at a time. At each iteration, a surrogate constraint is derived from a group of violated constraints, and the current point is orthogonally projected onto this surrogate constraint. The process is repeated until a feasible solution is found.

Yang and Murty [35] proposed three variants of surrogate constraint methods (see Section 3 for an overview). The $\mathrm{Ba}$ sic Surrogate Constraint Method (BSCM) takes all violated constraints in the system and makes successive projections of the current point. The Sequential Surrogate Constraint Method (SSCM) and the Parallel Surrogate Constraint Method (PSCM) on the other hand, work on a small subset of the violated constraints. SSCM is based on successive block projections, while PSCM is based on simultaneous block projections. Although the original PSCM converges slowly compared to SSCM, it becomes competitive with an adjusted step sizing rule [30].

Since BSCM has been shown to be faster than SSCM [30], we give efficient parallelizations of BSCM and the improved version of PSCM. State-of-the-art partitioning strategies are employed in the present paper for the parallelization of these two methods. Both BSCM and PSCM involve repeated matrixvector and matrix-transpose-vector multiplies, and regular operations such as inner products and vector updates. Partitioning sparse matrices is a crucial issue in the parallelization of BSCM and PSCM, since matrix-vector multiplies incur irregular dependencies. Both one-dimensional (1D), e.g., rowwise, and two-dimensional (2D), e.g., checkerboard, sparse matrix partitioning techniques are investigated (Sections 4 and 5, respectively). The recently proposed hypergraph partitioning models $[3,4,6,34]$ are used for load balancing and communication overhead minimization for both partitioning frameworks. Parallel algorithms for BSCM and PSCM are implemented for a message-passing multicomputer based on the above mentioned partitioning frameworks. The performance of the parallel implementations, the effects of different partitioning strategies on the parallel performance and on the speed of convergence, and the restoration abilities of the surrogate constraint methods are investigated experimentally in Section 6.

\section{Background}

\subsection{Formulation of the problem}

A general formulation of the image restoration problem with nonseparable, anisotropic, space variant and nonlocal distortions is given in [31], where the image $g(r)$ recorded on the film of an image $f(\rho(r, t))$ is given by

$$
g(r)=c \int_{t=0}^{T_{r}} f(\rho(r, t)) \mathrm{d} t .
$$

Here, $t$ denotes time, $T_{r}$ denotes the duration of the recording period, $r$ denotes the position vector on the 2D image, $c$ is a constant, $f(\rho(r, t))$ represents the observed (distorted) image, $\rho(r, t)$ represents the time varying, nonlinear distortion which can model the following types of motion:

(1) Translational motion: $\rho(r, t)=r-r(t)$, where $\rho(r, t)$ is a given function representing the motion of the original image or camera as a function of time (arbitrary 2D motions and accelerations are possible);

(2) Isotropic scaling: $\rho(r, t)=r / m(t)$, where $m(t)$ is an arbitrary scaling function of time (by properly choosing $m(t)$, it is possible to model the movement of the object towards or away from the camera);

(3) Rotation: $\rho(r, t)=R_{\phi(t)} r$, where $R_{\phi(t)}$ is the $2 \times 2$ rotation matrix for $\phi(t)$ which is an arbitrary function of time representing the angle of rotation.

The image restoration problem consists of recovering $f$ from $g$. Since, Eq. (2) represents a linear relation between $g$ and $f$, it is possible to write it as

$$
g(r)=\int_{r^{\prime}} H\left(r, r^{\prime}\right) f\left(r^{\prime}\right) \mathrm{d} r^{\prime},
$$

where $H\left(r, r^{\prime}\right)$ represents the blurring system. The discrete counterpart of Eq. (3) is simply the linear system of equations $g=H f$, where $g$ and $f$ are $m n \times 1$ vectors and $H$ is an $m n \times m n$ matrix for an image of size $m \times n$.

Typically, there is a measurement error or noise associated with the observation $g$, leading to an inconsistent system of equations. Denoting the noisy observation by $g^{\prime}$, the problem can be expressed as a system of inequalities:

$$
\left|\left(g^{\prime}-H f\right)_{i}\right| \leqslant \varepsilon, \quad i=1, \ldots, m n,
$$

where $\left(g^{\prime}-H f\right)_{i}$ is the $i$ th component of $g^{\prime}-H f$, and $\varepsilon$ is a suitable error tolerance parameter which is usually taken as a percentage of the mean value of $g$. For $i=1, \ldots, m n$, $\left|\left(g^{\prime}-H f\right)_{i}\right| \leqslant \varepsilon$ implies that

$$
\begin{aligned}
H f_{i} \leqslant \varepsilon+g_{i}^{\prime} & \text { if } g_{i}^{\prime} \leqslant H f_{i}, \\
-H f_{i} \leqslant \varepsilon-g_{i}^{\prime} & \text { if } g_{i}^{\prime} \geqslant H f_{i} .
\end{aligned}
$$

Thus, the above system is converted to a linear feasibility problem of the form

$$
\begin{aligned}
& A x \leqslant b \quad \text { by setting } \\
& A=\left[\begin{array}{c}
H \\
-H
\end{array}\right]_{2 m n \times m n}, \quad x=f_{m n \times 1}, \quad b=\left[\begin{array}{c}
\varepsilon+g^{\prime} \\
\varepsilon-g^{\prime}
\end{array}\right]_{2 m n \times 1},
\end{aligned}
$$

where $\varepsilon$ is an $m n \times 1$ vector of $\varepsilon$ 's. For further details, the reader is referred to [29, Chapter 5]. 


\subsection{Parallel matrix-vector multiplies}

\subsubsection{Algorithms based on $1 D$ matrix partitioning}

Suppose that rows and columns of an $M \times N$ matrix $H$ are permuted to yield a $K \times K$ block structure as

$$
\left[\begin{array}{cccc}
H_{11} & H_{12} & \cdots & H_{1 K} \\
H_{21} & H_{22} & \cdots & H_{2 K} \\
\vdots & \vdots & \ddots & \vdots \\
H_{K 1} & H_{K 2} & \cdots & H_{K K}
\end{array}\right]
$$

for rowwise partitioning among $K$ processors. Each processor $P_{k}$ holds the $k$ th row-stripe $H_{k}=\left[H_{k 1} \cdots H_{k K}\right]$ of size $m_{k} \times N$, where $\sum m_{k}=M$. The $k$ th column-stripe $\left[H_{1 k}^{T} \cdots H_{K k}^{T}\right]^{T}$ is of size $M \times n_{k}$, where $\sum n_{k}=N$.

In row-parallel $y \leftarrow H x$ multiply, the $y$ and $x$ vectors are partitioned as $y=\left[y_{1}^{T} \cdots y_{K}^{T}\right]^{T}$ and $x=\left[x_{1}^{T} \cdots x_{K}^{T}\right]^{T}$, where processor $P_{k}$ is responsible for computing subvector $y_{k}$ of size $m_{k}$ while holding subvector $x_{k}$ of size $n_{k}$. In this setting, the common algorithm $[13,33,34]$ executes the following steps at each processor $P_{k}$ :

(1) For each nonzero off-diagonal block $H_{\ell k}$, send sparse vector $\hat{x}_{k}^{\ell}$ to processor $P_{\ell}$, where $\hat{x}_{k}^{\ell}$ contains only those entries of $x_{k}$ corresponding to the nonzero columns in $H_{\ell k}$. For each nonzero off-diagonal block $H_{k \ell}$, receive $\hat{x}_{\ell}^{k}$ from processor $P_{\ell}$.

(2) Perform the local matrix-vector multiply $y_{k} \leftarrow H_{k} \times \tilde{x}_{k}$, where $\tilde{x}_{k}$ is the union of the local $x_{k}$ vector and $\hat{x}_{\ell}^{k}$ subvectors received in step 1 .

In step $1, P_{k}$ might be sending the same $x_{k}$-vector entry to different processors according to the sparsity pattern of the respective column of $H$. This multicast-like operation is referred to as expand operation.

In column-parallel $q \leftarrow H^{T} \pi$ multiply, processor $P_{k}$ effectively stores $\left(H_{k}\right)^{T}=H_{k}^{T}$ which is the $k$ th column-stripe of $H^{T}$. The $\pi$ and $q$ vectors are partitioned as $\pi=\left[\pi_{1}^{T} \cdots \pi_{K}^{T}\right]^{T}$ and $q=\left[q_{1}^{T} \cdots q_{K}^{T}\right]^{T}$, where processor $P_{k}$ is responsible for computing subvector $q_{k}$ of size $n_{k}$ while holding subvector $\pi_{k}$ of size $m_{k}$. In this setting, each processor $P_{k}$ executes the following steps:

(1) Perform the local matrix-vector multiply $q_{k} \leftarrow H_{k}^{T} \pi_{k}$.

(2) For each nonzero off-diagonal block $H_{\ell k}^{T}$, form sparse vector $\hat{q}_{\ell}^{k}$ which contains only those results of $q_{\ell}^{k}=H_{\ell k}^{T} \times \pi_{k}$ corresponding to the nonzero rows in $H_{\ell k}^{T}$. Send $\hat{q}_{\ell}^{k}$ to processor $P_{\ell}$. For each nonzero off-diagonal block $H_{k \ell}^{T}$ receive partial result $\hat{q}_{k}^{\ell}$ from processor $P_{\ell}$, and update $q_{k} \leftarrow$ $q_{k}+\hat{q}_{k}^{\ell}$.

In step 2, the multinode accumulation performed on $q_{k}$-vector entries is referred to as fold operation.

\subsubsection{Algorithms based on $2 D$ matrix partitioning}

Consider a $2 \mathrm{D}$ checkerboard partitioning on $H$ for the computations of the form $y \leftarrow H x$. In this partitioning scheme, the rows and the columns of matrix $H$ are divided into $R$ row-stripes and $C$ column-stripes yielding an $R \times C$ block structure. This block structure generalizes the one given in Eq. (7), and can be mapped naturally onto a 2D mesh ( $R$ rows and $C$ columns) of $K=R \times C$ processors. Therefore, the parallel system is considered as a logical 2D mesh [20]. In this setting, block $H_{k \ell}$ is assigned to processor $P_{k \ell}$. Note that nonzeros in any row (column) of matrix $H$ are assigned to the processors in the same row (column) of the processor mesh.

For the sake of clarity, we define a two level partitioning on the vectors $x$ and $y$. In the first level, the row and column-stripes of the $H$ matrix define $R$ - and $C$-way partitions on the $y$ and $x$ vectors, respectively. In the second level, each $x$ and $y$ subvector is assumed to be further partitioned into $R$ and $C$ subsubvectors, respectively. For example, $y_{k}$ denotes the $k$ th subvector of $y$ which contains subsubvector $y_{k \ell}$ for $\ell=1, \ldots, C$. In a dual manner, $x_{\ell}$ denotes the $\ell$ th subvector of $x$ which contains $x_{k \ell}$ for $k=1, \ldots, R$. A dual scheme is adopted in indexing the $x$ and $y$ subsubvectors so that each processor holds the subsubvectors of $x$ and $y$ with the same indices.

In 2D row-column-parallel $y \leftarrow H x$ multiply, each processor $P_{k \ell}$ is responsible for computing the subsubvector $y_{k \ell}$ while holding subsubvector $x_{k \ell}$. In this setting, $C$ row-parallel submatrix-vector multiplies algorithms are concurrently performed along the columns of the processor mesh to compute $C$ partial result vectors $y^{\ell}$ for $\ell=1, \ldots, C$, where $y=\sum_{\ell} y^{\ell}$. That is, for each $\ell=1, \ldots, C$, all of the $R$ processors in the $\ell$ th column of the processor mesh execute the row-parallel algorithm for computing the submatrix-vector multiply $y^{\ell} \leftarrow$ $H_{* \ell} x_{\ell}$, where $H_{* \ell}$ denotes the $\ell$ th column-stripe of matrix $H$ in block structure. At the end of this step, processor $P_{k \ell}$ holds the $k$ th portion $y_{k}^{\ell}$ of $y^{\ell}$. Then, $R$ fold operations are concurrently executed along the rows of the processor mesh to compute $y \leftarrow \sum_{\ell} y^{\ell}$. That is, for each $k=1, \ldots, R$, all of the $C$ processors in the $k$ th row of the processor mesh perform multinode accumulation on the $y_{k}$-subvector entries so that $P_{k \ell}$ ends up with the subsubvector $y_{k \ell}$. This last step effectively corresponds to step 2 - fold communication step-of the column-parallel matrix-vector multiply algorithm $y_{k} \leftarrow H_{k *} x$, where $H_{k *}$ denotes the $k$ th row-stripe of matrix $H$ in block structure.

\section{Surrogate constraint methods}

The successive orthogonal projections method developed by Kacmarz (see [32]) successively projects iterate $x^{t}$ at iteration $t$ onto hyperplanes $a_{i} x^{t}=b_{i}$ corresponding to those inequalities violated by $x^{t}$, where $a_{i}$ denotes the $i$ th row of $A$. Surrogate constraint methods [35], on the other hand, derive surrogate hyperplanes from a set of violated constraints, and take the projection of the current point onto surrogate hyperplanes. Surrogate hyperplanes eliminate the drawback of making projections for each of the violated constraints. Among the methods proposed by Yang and Murty [35], the BSCM derives surrogate hyperplanes from all of the violated constraints in the system, whereas the SSCM and the PSCM consider a subset of the constraints. 


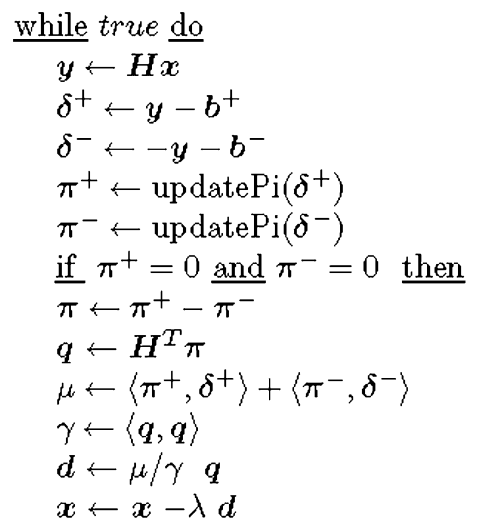

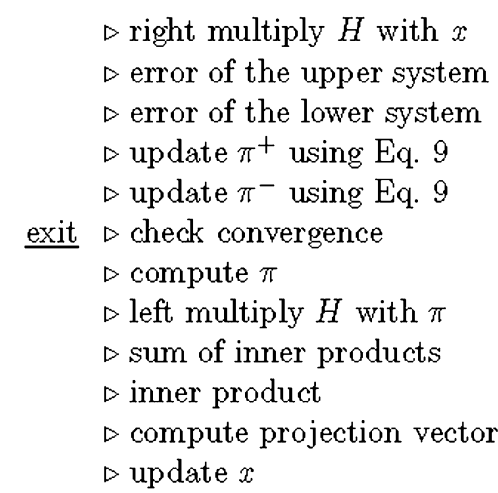

Fig. 1. BSCM applied to Eq. (6).

\subsection{Basic surrogate constraint method (BSCM)}

BSCM combines all of the violated constraints and makes just one projection at each iteration $t$ : if the current point $x^{t}$ violates the system $A x \leqslant b$, a $1 \times M$ weight vector $\pi$ is generated where $0<\pi_{i}<1$ if the $i$ th constraint is violated (i.e., $a_{i} x^{t}>$ $\left.b_{i}\right)$, and $\pi_{i}=0$ otherwise. The $\pi_{i}$ values are normalized so that $\sum_{i=1}^{M} \pi_{i}=1$ [35]. Then, the surrogate constraint $(\pi A) x^{t} \leqslant(\pi b)$ is generated for which the corresponding surrogate hyperplane is $S_{h}=\left\{x:(\pi A) x^{t}=(\pi b)\right\}$. The next point $x^{t+1}$ is obtained by projecting $x^{t}$ onto $S_{h}$ as

$$
x^{t+1}=x^{t}-\lambda d^{t} \quad \text { where } d^{t}=\frac{\pi A x^{t}-\pi b}{\|\pi A\|^{2}}(\pi A)^{T} .
$$

Here, $d^{t}$ is the projection vector, and $\lambda$ is a relaxation parameter that determines the location of the next point which is in the line segment joining the current point and its projection on the hyperplane. When $\lambda=1$ the next point is the exact orthogonal projection of the current point. If $0<\lambda<1$, the step taken is shorter (underrelaxation) and if $1<\lambda<2$, then the step taken is longer (overrelaxation) [8].

The selection of the weight vector $\pi$ is an issue for the solution of the problem. Weights may be distributed equally among all violated constraints or they can be assigned in proportion to the amount of violations. We use a hybrid approach to compute $\pi_{i}$ corresponding to the violated constraint $i$ as

$$
\pi_{i}=w_{1} \frac{\left(a_{i} x^{t}-b_{i}\right)}{\sum_{j \in V C}\left(a_{j} x^{t}-b_{j}\right)}+\frac{w_{2}}{|V C|},
$$

where $w_{1}$ and $w_{2}$ are two appropriate weights summing up to 1 , and $V C$ is the set of indices of the violated constraints at iteration $t$ [29].

Verification of the convergence of the algorithm is based on the strict Fejer-monotonicity of the generated sequence $\left\{x^{t}\right\}_{t=0}^{\infty}$, i.e., for any feasible $x$ and iteration $t$ it is true that $\left\|x^{t+1}-x\right\|<$ $\left\|x^{t}-x\right\|$. If the feasibility check allows a certain degree of tolerance $\varepsilon$, so that $A_{i} x^{t}$ is compared with $b_{i}+\varepsilon$, then the algorithm converges after a finite number of iterations [35].

Fig. 1 displays the pseudocode of BSCM applied to the system in Eq. (6). Since the system is composed of two copies of the same matrix with different signs, only the positive one is held during the computations. We call the system $H x \leqslant \varepsilon+g$ as the upper system and $-H x \leqslant \varepsilon-g$ as the lower system. Since $q=\pi^{+} H+\pi^{-}(-H)=\left(\pi^{+}-\pi^{-}\right) H$, we form the vector $\pi \leftarrow \pi^{+}-\pi^{-}$and then perform the multiply $\pi H$. To compute $y^{-} \leftarrow-H x$, simply $y \leftarrow H x$ is negated. As a result the sparse matrix vector multiplies $-H x$ and $\pi^{-}(-H)$ are avoided. Note that the original form of BSCM can be recovered by removing the operations involving vectors with a "-" superscript. In Fig. 1 and in the following pseudocodes, bold upper case letters denote matrices, bold lowercase letters denote column vectors of appropriate dimensions, plain lowercase letters denote scalars, and $\langle\cdot, \cdot\rangle$ denotes the inner product of two vectors.

The run time of a single iteration of BSCM applied to Eq. (6) is

$$
T_{\mathrm{BSCM}}=(4 Z+13 M+5 N) t_{\text {flop }},
$$

where $Z$ denotes the number of nonzeros in matrix $H$, and $t_{\text {flop }}$ denotes the time taken for a single scalar addition or multiplication operation.

\subsection{Sequential surrogate constraint method (SSCM)}

Instead of working on the entire $A$ matrix, SSCM partitions the system into subsystems and then solves the feasibility problem by applying the basic method on the subsystems in a cyclic order. Specifically, let matrix $A$ be partitioned rowwise into $K$ submatrices, and the right-hand side vector $b$ be partitioned conformably into $K$ subvectors, as follows:

$$
A=\left[\begin{array}{c}
A_{1} \\
\vdots \\
A_{k} \\
\vdots \\
A_{K}
\end{array}\right], \quad b=\left[\begin{array}{c}
b_{1} \\
\vdots \\
b_{k} \\
\vdots \\
b_{K}
\end{array}\right] .
$$

Here, $A_{k}$ is an $m_{k} \times N$ submatrix having $z_{k}$ nonzeros, $b_{k}$ is an $m_{k} \times 1$ subvector, and $\sum_{k=1}^{K} m_{k}=M$ and $\sum_{k=1}^{K} z_{k}=Z$. Surrogate constraints are defined as $\left(\pi_{k} A_{k}\right) x \leqslant \pi_{k} b_{k}$ for each block, where $\pi_{k}$ is of dimension $m_{k}$ and is as defined above. 


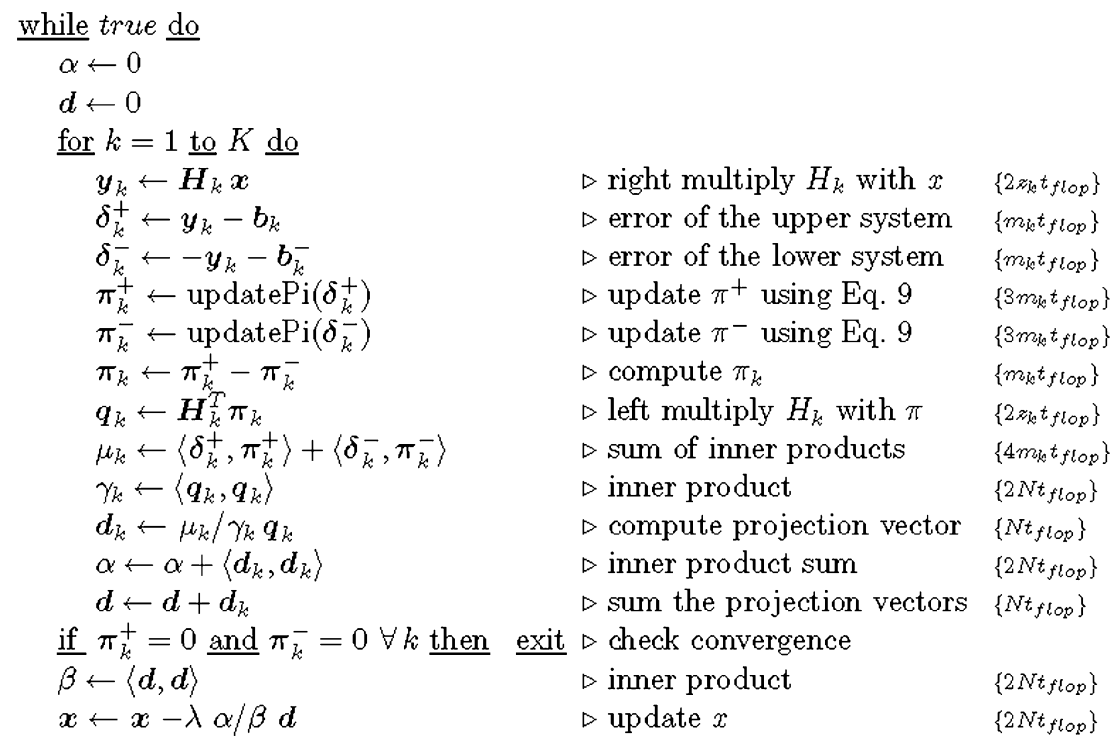

Fig. 2. PSCM applied to Eq. (6).

SSCM proceeds by projecting the current iterate onto surrogate hyperplanes $\left(\pi_{k} A_{k}\right) x=\pi_{k} b_{k}$ successively for $k=1, \ldots, K$ in cyclic order and increments $t$ at each block. For each block, the next point is computed as

$x^{t+1}=x^{t}-\lambda d_{k}^{t} \quad$ where $d_{k}^{t}=\frac{\pi_{k}^{t}\left(A_{k} x^{t}-b_{k}\right)}{\left\|\pi_{k}^{t} A_{k}\right\|^{2}}\left(\pi_{k}^{t} A_{k}\right)^{T}$.

Here, $d_{k}^{t}$ is the projection vector of the $k$ th block.

In SSCM each point $x^{t}$ that is projected on block $k$ is a result of the projection of $x^{t-1}$ performed in the preceding block $k-1$. Thus, successive block projections imply a dependency between the blocks of the system, causing the algorithm to be highly sequential.

The run time of SSCM applied to Eq. (6) is

$$
\begin{aligned}
T_{S S C M} & =\sum_{k=1}^{K}\left(4 z_{k}+13 m_{k}+5 N\right) t_{\text {flop }} \\
& =(4 Z+13 M+5 N K) t_{\text {flop }}
\end{aligned}
$$

Note that each block brings an extra computation time of $5 N t_{\text {flop. }}$.

\subsection{Parallel surrogate constraint method (PSCM)}

Yang and Murty [35] proposed PSCM as a coarse-grain parallel variant of SSCM which overcomes its serial nature. PSCM carries out simultaneous block projections and generates the next point as a convex combination of these projections. As in SSCM, $A$ is divided rowwise into $K$ contiguous blocks as shown in Eq. (11). At iteration $t$ of PSCM, the next point $x^{t+1}$ is computed as

$$
x^{t+1}=x^{t}-\lambda \sum_{k=1}^{K} \tau_{k} d_{k}^{t}
$$

Here, $\tau_{k}$ are nonnegative numbers summing up to 1 , and $d_{k}^{t}$ is as defined in Eq. (12).

In Eq. (14), each projection has its own influence $\tau_{k}$. This influence is taken into account to accelerate the convergence. Hence, $\tau_{k}$ can be taken to be proportional to the number of violated constraints or the cumulative error of the respective block. However, as clarified in [29], no matter how $\tau_{k}$ is chosen, the progress of PSCM is much slower than that of SSCM. Actually this method is a variant of the Cimmino type algorithms which are known to suffer from slow convergence. To alleviate this problem, García-Palomares and Gonzales-Castaño [9] proposed an acceleration procedure for the Cimmino type algorithms by giving an improved step sizing rule. Later, Özaktaş et al. [30] used this rule in the parallel surrogate algorithm and generated the next point as follows:

$$
x^{t+1}=x^{t}-\lambda \frac{\sum_{k=1}^{K}\left\|d_{k}^{t}\right\|^{2}}{\left\|\sum_{k=1}^{K} d_{k}^{t}\right\|^{2}} \sum_{k=1}^{K} d_{k}^{t},
$$

where $d_{k}^{t}$ is defined as in Eq. (12). With this modification, the step sizes taken are enlarged so that the parallel method converges much more rapidly. Fig. 2 displays PSCM applied to Eq. (6).

The run time of PSCM applied to Eq. (6) is

$$
\begin{aligned}
T_{\text {PSCM }} & =\sum_{k=1}^{K}\left(4 z_{k}+13 m_{k}+6 N\right) t_{\text {flop }}+4 N t_{\text {flop }} \\
& =(4 Z+13 M+6 N K+4 N) t_{\text {flop }} .
\end{aligned}
$$

For SSCM and improved PSCM, as the number of blocks, $K$, increases, the number of iterations required for convergence is likely to decrease. However, each block brings an extra run time of $5 N t_{\text {flop }}$ and $6 N t_{\text {flop }}$ for SSCM and PSCM, respectively. 
Hence, the run times of SSCM and PSCM may increase by the increasing $K$, especially for highly sparse systems.

\section{Parallelization based on 1D matrix partitioning}

As seen in Figs. 1 and 2, repeated matrix-vector and matrixtranspose-vector multiplies of the forms $y \leftarrow H x$ and $q \leftarrow$ $H^{T} \pi$ constitute the computational kernels of both BSCM and PSCM. These methods also involve linear vector operations such as inner products and vector updates. In terms of the dependencies between matrix-vector multiplies and linear vector operations, the vectors can be classified as $x$-space and $y$-space vectors so that the linear vector operations occur only between the vectors that are in the same space. In this setting, $x, q$, and $d$ are $x$-space vectors, whereas $y, b^{+}, b^{-}, \pi, \pi^{+}, \pi^{-}, \delta^{+}$, and $\delta^{-}$are $y$-space vectors.

In 1D parallelization of BSCM, the matrix $H$ may be partitioned rowwise or columnwise. However, since the PSCM formulation is based on rowwise blocking, it requires rowwise partitioning of matrix $H$. Thus, for the sake of simplicity, we assume a $K$-way rowwise partitioning of $H$ for both methods, where $K$ denotes the number of processors. A rowwise partition on $H$ induces a columnwise partition on $H^{T}$. Therefore, the row-parallel algorithm is adopted for $y \leftarrow H x$, and the column-parallel algorithm is adopted for $q \leftarrow H^{T} \pi$.

\subsection{Basic surrogate constraint method}

Parallel BSCM based on 1D partitioning executes the steps given in Fig. 3 at each processor $P_{k}$. In this figure and the following figures, subscripts are used to denote the subvectors and submatrices that are stored locally in a processor, whereas superscripts are used to denote the partial results computed by a processor. For example, $H_{k}$ denotes the $k$ th row-stripe of the global $H$ matrix stored by the processor $P_{k} ; x_{k}$ denotes the $k$ th stripe of the global $x$ vector maintained by processor $P_{k} ; \gamma^{k} \leftarrow$ $\left\langle q_{k}, q_{k}\right\rangle$ denotes partial inner product result for the global inner product $\gamma \leftarrow\langle q, q\rangle$ computed by processor $P_{k}$. The global communication operators globalAnd and globalSum combine the input arguments of each processor using the operations and and sum respectively, and distribute the result back to all processors. As seen in Fig. 3, scalars $\mu$ and $\gamma$ are reduced together in order to amortize the latency overhead in the reduction operations.

\subsection{Parallel surrogate constraint method}

Let the number of blocks, $K$, be equivalent to the number of processors, that is each processor is given a single block. Then, parallel PSCM executes the steps given in Fig. 4 at each processor $P_{k}$. Since the resulting vector $q$ for $q \leftarrow H^{T} \pi$ multiply need not to be constructed, the parallelization of PSCM does not necessitate the execution of the column-parallel multiply algorithm as a whole. As seen in the figure, the local projection vector $d^{k}$ is obtained from the local intermediate vector $q^{k}$ through linear vector operations, and then the sum of the local projection vectors is computed through a fold operation on these local $d^{k}$ vectors. This fold operation on the local $d^{k}$ vectors can be considered as the delayed execution of the fold operation in step 2 of the column-parallel multiply algorithm. Note that this fold operation also provides each processor $P_{k}$ with the subvector $d_{k}$ needed to update $x_{k}$. The scalar $\alpha$ is obtained through reducing the inner products of local projection vectors $\alpha^{k}=\left\|d^{k}\right\|^{2}$ using the globalSum operator. The scalar $\beta$ is obtained through computing the inner product of global projection vector $d$. Computing $\beta$ in parallel requires reducing the $\beta^{k}=\left\|d_{k}\right\|^{2}$ values. The scalars $\alpha$ and $\beta$ are reduced together for efficiency issues as in parallel BSCM.

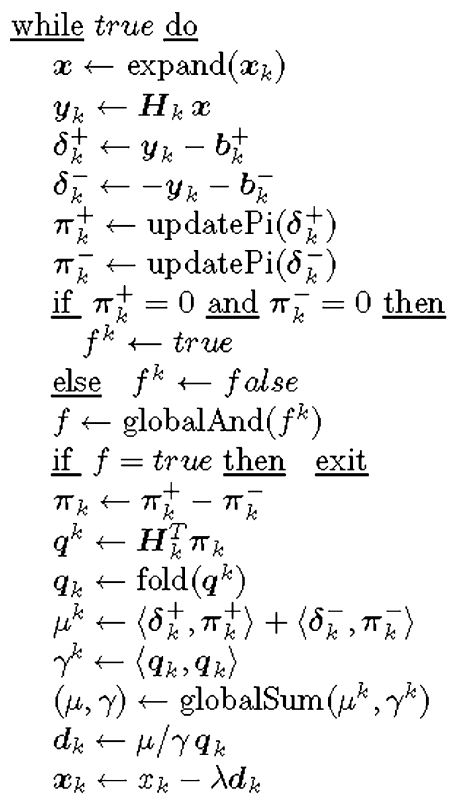

$\begin{array}{ll}\triangleright \text { expand } x_{k} \text { vector } & \left\{t_{\text {expand }}\right\} \\ \triangleright \text { multiply } H_{k} \text { from right } & \left\{2 z_{k} t_{f l o p}\right\} \\ \triangleright \text { error of the upper system } & \left\{m_{k} t_{f l o p}\right\} \\ \triangleright \text { error of the lower system } & \left\{m_{k} t_{f l o p}\right\} \\ \triangleright \text { update } \pi^{+} \text {using Eq. } 9 & \left\{3 m_{k} t_{f l o p}\right\} \\ \triangleright \text { update } \pi^{-} \text {using Eq. } 9 & \left\{3 m_{k} t_{f l o p}\right\} \\ \triangleright \text { check convergence } \\ \triangleright \text { block } k \text { feasible } \\ \triangleright \text { block } k \text { not feasible } \\ \triangleright \text { check the whole system } \\ \\ \triangleright \text { compute } \pi_{k} \\ \triangleright \text { multiply } H_{k} \text { from left } & \left\{\left(t_{s}+t_{w}\right) \log K\right\} \\ \triangleright \text { fold } q \text { vector } & \left\{m_{k} t_{f l o p}\right\} \\ \triangleright \text { sum of inner products } & \left\{2 z_{k} t_{f l o p}\right\} \\ \triangleright \text { inner product } & \left\{4 m_{f o l}\right\} \\ \triangleright \text { form } \mu \text { and } \gamma & \left\{2 n_{k} t_{f l o p}\right\} \\ \triangleright \text { form projection vector } & \left\{\left(t_{s}+2 t_{w}\right) \log K\right\} \\ \triangleright \text { update my portion of } x & \left\{n_{k} t_{f l o p}\right\} \\ & \left\{2 n_{k} t_{f l o p}\right\}\end{array}$

Fig. 3. Parallel BSCM based on 1D partitioning of the $H$ matrix. 

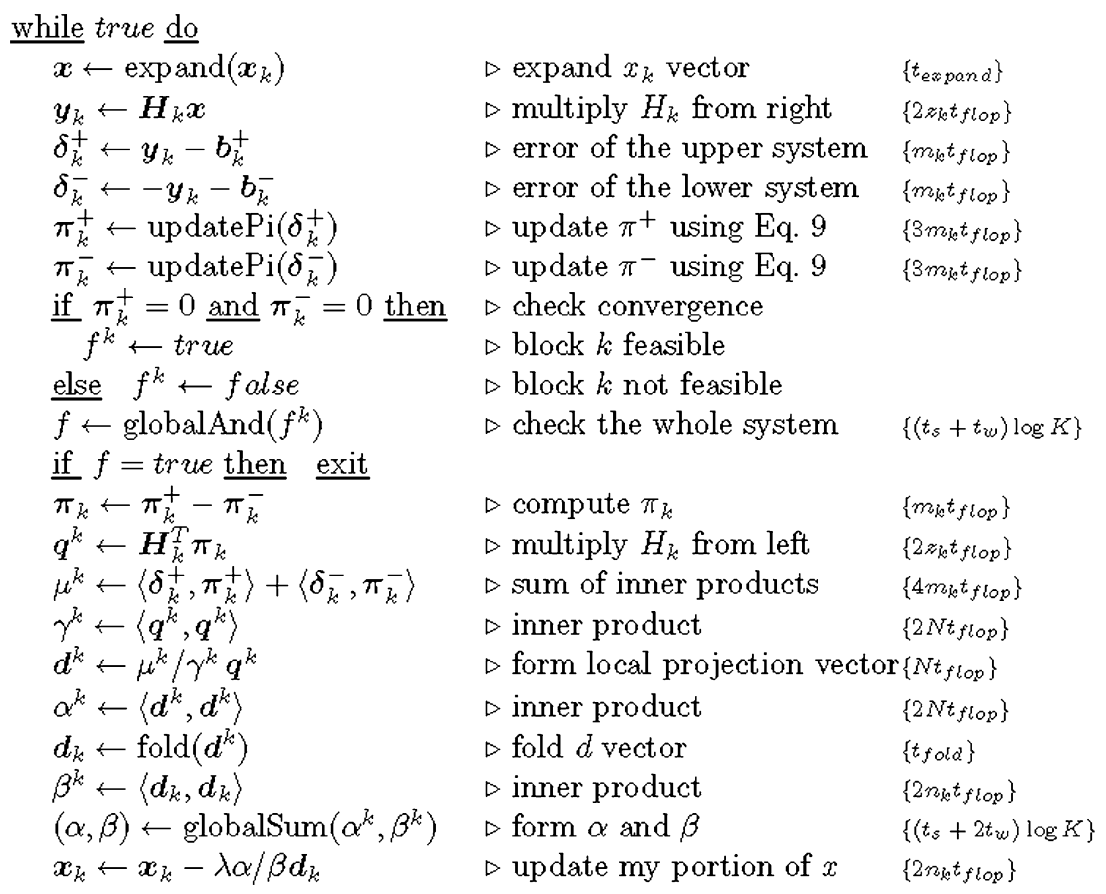

Fig. 4. Parallel PSCM based on 1D partitioning of the $H$ matrix.

\subsection{Load balancing and communication-overhead minimization}

Sparse matrix partitioning for parallel matrix-vector multiplies of the form $y \leftarrow H x$ is formulated in terms of the graph [17] and hypergraph partitioning problems [4]. Both of these problems are NP-complete [10,23]. We use the hypergraphbased formulation for two reasons. First, the objective in hypergraph-based formulation is an exact measure of the total communication volume. Second, the matrices in our methods are unsymmetric; standard graph partitioning is not readily applicable $[4,13,14]$.

We use the column-net hypergraph model of Çatalyürek and Aykanat [4] to obtain a $K$-way rowwise partition on matrix $H$. The rowwise partition on $H$ induces a columnwise partition on $H^{T}$. As is known $[13,34]$, the communication requirements of the row-parallel $y \leftarrow H x$ and the column-parallel $q \leftarrow H^{T} \pi$ multiplies are the same in terms of the total volume and number of messages. Therefore, the above partitioning enables efficient multiplies with $H^{T}$ as well. As mentioned earlier, the $x$ and $y$-space vectors do not undergo linear vector operations. Hence, an unsymmetric partitioning on $H$ is allowable. We use the techniques discussed in [34] to exploit this freedom in order to minimize the communication overhead due to the total number of messages, maximum volume and number of messages handled by a single processor as well as the total volume of messages.

In parallelizing BSCM and PSCM, we consider balancing the computational loads of the processors only for the matrixvector and matrix-transpose-vector multiplies. The loads of the processors during linear vector operations are determined by the partitioning on $H$. Therefore, imbalances in processors' loads may occur during these operations. Obtaining balance in the vector operations is possible within multi-constraint partitioning framework $[5,18,19]$. In this work, we omit obtaining computational balance during linear vector operations for two reasons. First, imbalances in linear vector operations are tolerable, because these operations are not costly. Second, multiconstraint formulation restricts the search space in a hypergraph partitioning problem; this restriction may lead to a higher communication cost.

\section{Parallelization based on 2D matrix partitioning}

The row-column-parallel algorithm is used for both the matrix-vector multiply $y \leftarrow H x$ and matrix-transpose-vector multiply $q \leftarrow H^{T} \pi$. There is a duality between the interprocessor communication patterns of $y \leftarrow H x$ and $q \leftarrow H^{T} \pi$ multiplies. The communication pattern of the expand operation in $q \leftarrow H^{T} \pi$ is exactly the same as that of the fold operation in $y \leftarrow H x$ and vice versa.

\subsection{Basic surrogate constraint method}

Parallel BSCM based on checkerboard partitioning executes the steps given in Fig. 5 at each processor $P_{k \ell}$. We use the same convention on the usage of subscripts and superscripts. This time, however, we use two indices $k \ell$ to designate processor $P_{k \ell}$ 's data. The single indices $k$ or $\ell$ are used for the results pertaining to the $k$ th row-stripe or $\ell$ th column-stripe, respectively. Recall that the communication operations required for matrix-vector multiplies are confined to the rows or columns of the processor mesh. Only the scalars $\mu$ and $\gamma$ are obtained via a global sum operation among all processors. 
while true do

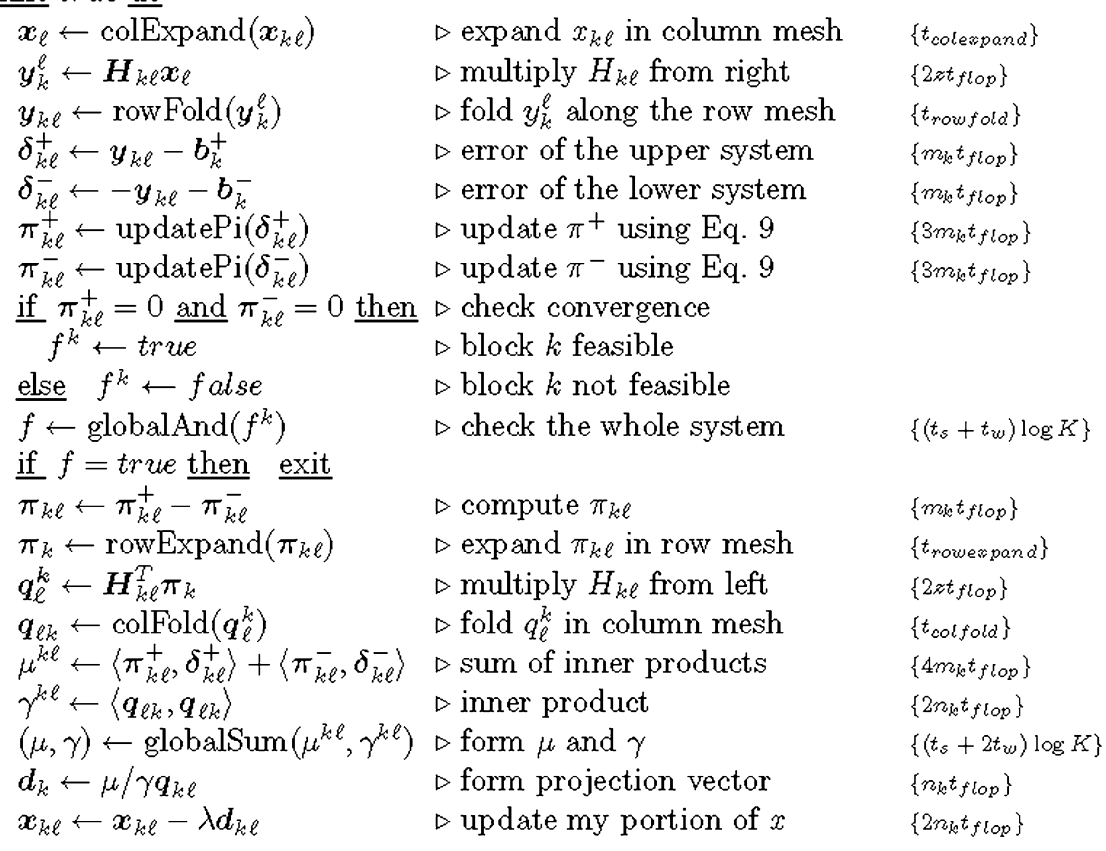

Fig. 5. Parallel BSCM based on 2D checkerboard partitioning of the $H$ matrix.

while true do

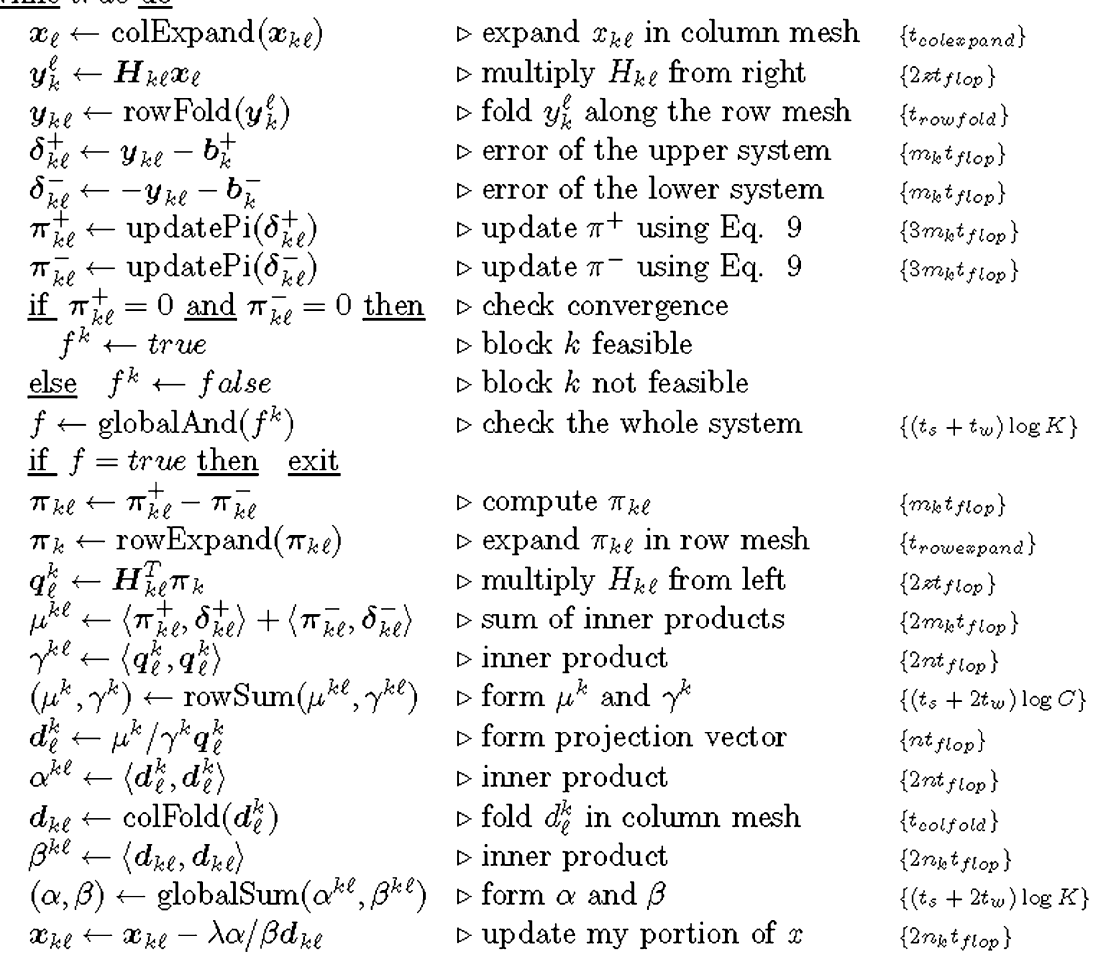

Fig. 6. Parallel PSCM based on 2D checkerboard partitioning of the $H$ matrix.

\subsection{Parallel surrogate constraint method}

Different from the 1D partitioning, the number of row blocks for PSCM is $R$ not $K$. Parallel PSCM based on checkerboard partitioning executes the steps given in Fig. 6 at each processor
$P_{k \ell}$. As seen in Fig. 6, the 2D implementation of PSCM is similar to the 2D implementation of BSCM. However, in order to calculate the projection vector of the $k$ th row-stripe, local $\mu^{k \ell}$ and $\gamma^{k \ell}$ scalars are computed and are added up in the $k$ th row of the processor mesh. Since $\alpha=\sum_{k=1}^{R} \sum_{\ell=1}^{C} \alpha^{k \ell}$ and 
$\beta=\sum_{k=1}^{R} \sum_{\ell=1}^{C} \beta^{k \ell}$, a global sum operation is required to apply the step sizing rule.

\subsection{Load balancing and communication-overhead minimization}

A number of different techniques for checkerboard partitioning of sparse matrices are given in $[6,15,25,24]$. Among these, only the hypergraph partitioning model of Çatalyürek and Aykanat [6] exploits sparsity to reduce communication cost. Therefore, we use hypergraph partitioning model to obtain an $R \times C$ checkerboard partition on the matrix $H$.

Since the communication operations are confined to the rows and columns of the processor mesh, the maximum number of messages handled by a single processor for a parallel system with $K=R \times C$ processors is at most $R+C-2$ (compared to $K-1$ in 1D). Therefore, the checkerboard partitioning method leads to reduced communication latency overhead without explicit effort.

The row-column-parallel matrix-vector multiply algorithm given in Section 2.2.2 uses point-to-point (personalized) communication scheme for the expand and fold operations. Since the expand and fold operations are confined to a smaller number of processors in 2D checkerboard partitioning, all-to-all communication schemes are viable, i.e., an all-to-all broadcast and a multinode accumulation can be performed for the expand and fold operations. These algorithms can be implemented using hypercube algorithms to reduce the maximum number of messages handled by a processor to $\lceil\log R\rceil+\lceil\log C\rceil \approx\lceil\log K\rceil$. The 2D hypergraph model [6] can be extended to handle the minimization of communication volume overhead in this allto-all communication scheme [2].

\section{Results}

The parallel performance and the restoration abilities of the surrogate constraint methods are evaluated experimentally. Parallel performance analysis is first carried on an iteration basis. This approach shows the amount of gain achieved by the
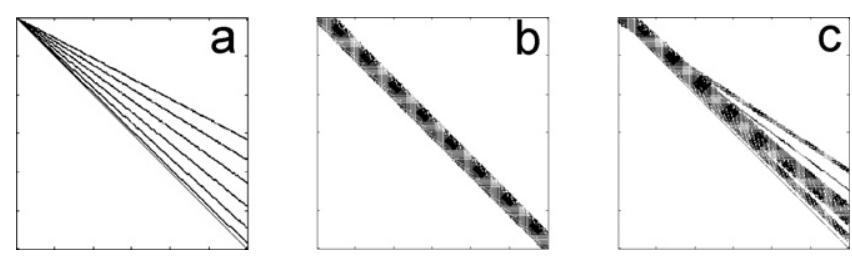

Fig. 7. Sparsity patterns of the $H$ matrices corresponding to the three types of blur: (a) iso $150 \times 200$; (b) $\operatorname{rot} 150 \times 200$; (c) irt150 $\times 200$.

proposed parallelization schemes. Then, overall performance results and examples of blurred and restored images are given.

The experiments were carried out on a Beowulf Cluster equipped with $400 \mathrm{MHz}$ Intel Pentium II processors with $512 \mathrm{~KB}$ cache size and $128 \mathrm{MB}$ RAM. The operating system is Debian GNU/Linux 3.0 distribution with Linux kernel 2.4.14. The interconnection network is comprised of a $3 \mathrm{COM} \mathrm{Su}$ perStack II 3900 managed switch connected to Intel Ethernet Pro 100 Fast Ethernet network interface cards at each node. The parallel algorithms were implemented using LAM/MPI 6.5.6 [1].

\subsection{Experimental setup}

Three types of blurs were used for the construction of the distorted images. In all of the blurs, the record time was set as $3.5 \mathrm{~s}$ and the movement of the object is sampled at $0.5 \mathrm{~s}$ intervals. The first type of blur models isotropic scaling. The $x$ - and $y$-axis coordinates of the function $\rho$ were chosen as $x /\left(1+0.1 s^{2}\right)$ and $y /\left(1+0.1 s^{2}\right)$. The second blur is a result of rotation motion. The object was rotated clockwise $6^{\circ}$ per second for the first $1.5 \mathrm{~s}$ and then was rotated counter-clockwise $4^{\circ}$ per second for the remaining $2 \mathrm{~s}$. The third blur denotes a combined effect of translational motion, isotropic scaling, and rotation. In the translational motion, the object accelerates with $0.5 \mathrm{~m} / \mathrm{s}^{2}$ in the $x$ direction for the first $1.5 \mathrm{~s}$ and then it turns back with a constant speed of $1.0 \mathrm{~m} / \mathrm{s}$, and moves along the $y$ direction with a constant speed of $1.0 \mathrm{~m} / \mathrm{s}$ throughout the recording time. Isotropic scaling and rotation effects of this blur are the same as those of the first and second blurs, respectively.

Table 1

Properties of the $H$ matrices

\begin{tabular}{|c|c|c|c|c|c|c|c|}
\hline \multirow[t]{4}{*}{$H$ matrix } & \multirow[t]{4}{*}{ Number of rows/cols } & \multicolumn{6}{|c|}{ Number of nonzeros } \\
\hline & & \multirow[t]{3}{*}{ Total } & \multicolumn{5}{|l|}{ Per } \\
\hline & & & \multirow{2}{*}{$\begin{array}{l}\text { Row/col } \\
\text { Avg }\end{array}$} & \multicolumn{2}{|l|}{ Row } & \multicolumn{2}{|l|}{ Col } \\
\hline & & & & $\operatorname{Min}$ & $\overline{\operatorname{Max}}$ & Min & $\operatorname{Max}$ \\
\hline iso $150 \times 200$ & 30000 & 209377 & 6.98 & 1 & 16 & 1 & 7 \\
\hline iso $300 \times 400$ & 120000 & 839377 & 6.99 & 1 & 16 & 1 & 7 \\
\hline iso600 $\times 800$ & 480000 & 3359377 & 7.00 & 1 & 16 & 1 & 7 \\
\hline $\operatorname{rot} 150 \times 200$ & 30000 & 168775 & 5.63 & 1 & 8 & 1 & 6 \\
\hline $\operatorname{rot} 300 \times 400$ & 120000 & 681907 & 5.68 & 1 & 8 & 1 & 6 \\
\hline $\operatorname{rot} 600 \times 800$ & 480000 & 2734319 & 5.70 & 1 & 8 & 1 & 6 \\
\hline $\operatorname{irt} 150 \times 200$ & 30000 & 205633 & 6.85 & 1 & 19 & 3 & 7 \\
\hline $\operatorname{irt} 300 \times 400$ & 120000 & 823661 & 6.86 & 1 & 19 & 3 & 7 \\
\hline $\operatorname{irt600\times 800~}$ & 480000 & 3294639 & 6.86 & 1 & 19 & 3 & 7 \\
\hline
\end{tabular}


Table 2

Per iteration execution times of parallel BSCM and PSCM (in ms)

\begin{tabular}{|c|c|c|c|c|c|c|c|c|}
\hline \multirow[t]{3}{*}{$H$ matrix } & \multirow[t]{3}{*}{ Sequential BSCM } & \multirow[t]{3}{*}{$K$} & \multicolumn{3}{|c|}{ Parallel BSCM } & \multicolumn{3}{|c|}{ Parallel PSCM } \\
\hline & & & \multirow{2}{*}{$\begin{array}{l}1 \mathrm{D} \\
\mathrm{P} 2 \mathrm{P}\end{array}$} & \multicolumn{2}{|l|}{$2 \mathrm{D}$} & \multirow{2}{*}{$\begin{array}{l}\text { 1D } \\
\text { P2P }\end{array}$} & \multicolumn{2}{|l|}{$2 \mathrm{D}$} \\
\hline & & & & $\mathrm{P} 2 \mathrm{P}$ & $\mathrm{A} 2 \mathrm{~A}$ & & $\mathrm{P} 2 \mathrm{P}$ & $\mathrm{A} 2 \mathrm{~A}$ \\
\hline \multirow[t]{4}{*}{ iso $150 \times 200$} & 75.1 & 4 & 19.7 & 15.9 & 17.1 & 19.7 & 16.5 & 17.3 \\
\hline & & 8 & 11.3 & 9.8 & 11.2 & 11.2 & 10.1 & 10.5 \\
\hline & & 16 & 7.3 & 6.9 & 9.6 & 7.1 & 7.1 & 8.0 \\
\hline & & 24 & 6.0 & 6.3 & 10.5 & 6.0 & 6.5 & 8.0 \\
\hline \multirow{4}{*}{ iso300 $\times 400$} & 324.1 & 4 & 81.1 & 67.7 & 69.4 & 81.8 & 70.2 & 70.1 \\
\hline & & 8 & 41.3 & 34.2 & 37.9 & 41.2 & 35.0 & 36.6 \\
\hline & & 16 & 22.3 & 19.7 & 24.8 & 22.3 & 20.0 & 22.3 \\
\hline & & 24 & 16.5 & 15.4 & 23.2 & 16.7 & 15.6 & 19.0 \\
\hline \multirow[t]{4}{*}{ iso600 $\times 800$} & 1430.1 & 4 & 335.4 & 281.8 & 288.8 & 343.4 & 294.9 & 295.1 \\
\hline & & 8 & 171.8 & 141.5 & 149.0 & 175.0 & 146.8 & 149.3 \\
\hline & & 16 & 86.5 & 73.5 & 85.1 & 87.6 & 76.5 & 81.3 \\
\hline & & 24 & 60.1 & 51.6 & 64.7 & 60.5 & 52.8 & 59.0 \\
\hline \multirow[t]{4}{*}{$\operatorname{rot} 150 \times 200$} & 71.2 & & 17.9 & 14.6 & 15.6 & 18.0 & 15.4 & 16.0 \\
\hline & & 8 & 10.0 & 8.7 & 9.8 & 10.1 & 9.0 & 9.5 \\
\hline & & 16 & 6.3 & 6.1 & 8.0 & 6.4 & 6.3 & 6.9 \\
\hline & & 24 & 5.3 & 5.7 & 8.5 & 5.3 & 6.0 & 6.7 \\
\hline \multirow[t]{4}{*}{$\operatorname{rot} 300 \times 400$} & 299.5 & 4 & 75.9 & 63.0 & 65.1 & 76.9 & 65.4 & 66.0 \\
\hline & & 8 & 38.4 & 32.0 & 34.3 & 38.9 & 33.0 & 34.0 \\
\hline & & 16 & 20.4 & 17.9 & 21.1 & 20.6 & 18.3 & 19.6 \\
\hline & & 24 & 14.8 & 13.7 & 18.1 & 14.8 & 14.3 & 15.8 \\
\hline \multirow[t]{4}{*}{$\operatorname{rot} 600 \times 800$} & 1319.3 & 4 & 320.8 & 255.9 & 269.2 & 329.7 & 269.6 & 275.0 \\
\hline & & 8 & 164.7 & 132.5 & 138.7 & 169.1 & 138.0 & 140.9 \\
\hline & & 16 & 85.1 & 69.1 & 74.9 & 86.2 & 71.9 & 74.3 \\
\hline & & 24 & 56.2 & 47.8 & 56.1 & 56.7 & 49.6 & 53.1 \\
\hline \multirow[t]{4}{*}{$\operatorname{irt} 150 \times 200$} & 75.6 & 4 & 25.2 & 20.7 & 22.4 & 25.3 & 21.1 & 21.9 \\
\hline & & 8 & 17.3 & 15.1 & 23.0 & 17.5 & 15.5 & 18.3 \\
\hline & & 16 & 12.8 & 11.7 & 21.6 & 13.0 & 12.2 & 15.2 \\
\hline & & 24 & 10.9 & 9.8 & 23.0 & 10.9 & 10.2 & 15.7 \\
\hline \multirow[t]{4}{*}{$\operatorname{rot} 300 \times 400$} & 325.1 & 4 & 106.3 & 89.5 & 94.7 & 107.5 & 90.1 & 92.5 \\
\hline & & 8 & 84.8 & 59.6 & 85.4 & 86.1 & 60.7 & 74.8 \\
\hline & & 16 & 63.0 & 44.6 & 73.0 & 63.3 & 45.8 & 53.9 \\
\hline & & 24 & 43.9 & 32.1 & 74.1 & 44.3 & 33.5 & 51.6 \\
\hline \multirow[t]{4}{*}{$\operatorname{rot} 600 \times 800$} & 1419.7 & 4 & 508.6 & 407.8 & 412.8 & 518.5 & 418.5 & 416.3 \\
\hline & & 8 & 351.7 & 291.1 & 383.0 & 356.8 & 294.7 & 363.8 \\
\hline & & 16 & 274.7 & 171.1 & 301.4 & 277.3 & 171.0 & 259.8 \\
\hline & & 24 & 246.5 & 130.7 & 274.6 & 245.8 & 129.7 & 224.4 \\
\hline \multirow[t]{4}{*}{ Average speedup } & & 4 & 3.7 & 4.5 & 4.3 & 3.6 & 4.3 & 4.2 \\
\hline & & 8 & 6.4 & 7.8 & 6.8 & 6.4 & 7.6 & 7.1 \\
\hline & & 16 & 11.0 & 12.9 & 10.1 & 11.0 & 12.6 & 11.2 \\
\hline & & 24 & 14.8 & 16.8 & 11.6 & 14.7 & 16.3 & 13.6 \\
\hline
\end{tabular}

Using these blurring effects, three different images of $150 \times 200,300 \times 400$, and $600 \times 800$ pixels were produced with zero boundary condition, i.e., pixels outside the borders of the images are black [28]. Noise was simulated by adding $\% 1$ normally distributed zero mean random variables with a standard deviation of one, e.g., normally distributed random noise scaled such that 2-norm of the noise is 0.01 times the 2-norm of the blurred image. This type of noise is typical in similar works (see [27,26,28] and the references therein). Table 1 and Fig. 7 display the properties and the sparsity patterns of the resulting $H$ matrices. The prefixes "iso", "rot", and "irt" are, respectively, used to denote the isotropic, rotation, and combined (isotropic + rotation + translational) blurs. 

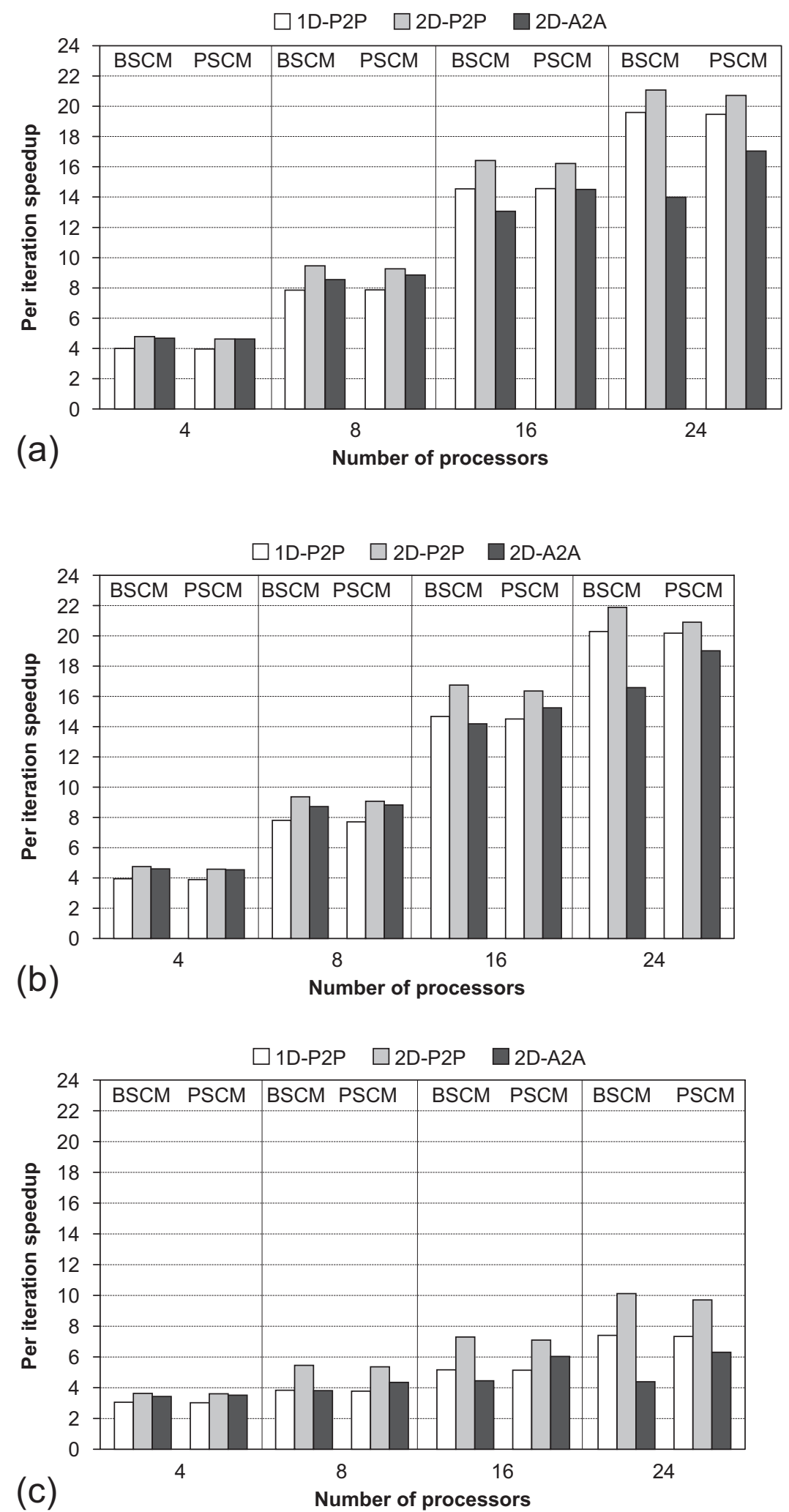

Fig. 8. Per iteration speedup charts of BSCM and PSCM for the images of size $300 \times 400$ : (a) isotropic blur; (b) rotation blur; (c) combined blur.

The hypergraph partitioning tool $\mathrm{PaToH}$ [5] was used with the default parameters to obtain the desired 1D and 2D partitionings. Since $\mathrm{PaToH}$ incorporates randomized algorithms, it was run 10 times starting from different seeds for every partitioning instance. In all partitioning instances, the observed imbalance ratios were below 5\%. Averages of the parallel 
Table 3

Communication patterns for the $H$ matrices corresponding to the images of size $300 \times 400$

\begin{tabular}{|c|c|c|c|c|c|c|c|}
\hline \multirow[t]{3}{*}{$H$ matrix } & \multirow[t]{3}{*}{$K$} & \multicolumn{2}{|c|}{ Total message } & \multicolumn{4}{|c|}{ Maximum message } \\
\hline & & Volume & Number & \multirow{2}{*}{$\begin{array}{l}\text { Volume } \\
y=H x\end{array}$} & Number & \multirow{2}{*}{$\begin{array}{l}\text { Volume } \\
q=H^{T} \pi\end{array}$} & \multirow{2}{*}{ Number } \\
\hline & & \multicolumn{2}{|c|}{$y=H x / q=H^{T} \pi$} & & & & \\
\hline \multicolumn{8}{|l|}{$1 D-P 2 P$} \\
\hline \multirow[t]{4}{*}{ iso300 $\times 400$} & 4 & 1721 & 7.4 & 560 & 2.5 & 702 & 2.8 \\
\hline & 8 & 3682 & 21.2 & 654 & 4.2 & 803 & 4.6 \\
\hline & 16 & 7794 & 48.5 & 703 & 6.5 & 859 & 5.4 \\
\hline & 24 & 12539 & 76.9 & 745 & 7.5 & 962 & 6.1 \\
\hline \multirow[t]{4}{*}{$\operatorname{rot} 300 \times 400$} & 4 & 598 & 6.2 & 187 & 2.1 & 248 & 2.1 \\
\hline & 8 & 1452 & 16.0 & 260 & 3.3 & 306 & 3.2 \\
\hline & 16 & 3159 & 35.2 & 297 & 4.2 & 333 & 3.6 \\
\hline & 24 & 5451 & 59.4 & 317 & 5.1 & 400 & 4.7 \\
\hline \multirow[t]{4}{*}{$\operatorname{irt} 300 \times 400$} & 4 & 33827 & 6.4 & 11089 & 2.2 & 15417 & 3.0 \\
\hline & 8 & 100993 & 29.8 & 15086 & 5.8 & 18833 & 5.8 \\
\hline & 16 & 163814 & 104.7 & 11579 & 10.4 & 14327 & 10.3 \\
\hline & 24 & 180096 & 168.3 & 8777 & 15.0 & 10904 & 11.3 \\
\hline \multicolumn{8}{|l|}{$2 D-P 2 P$} \\
\hline \multirow[t]{4}{*}{ iso300 $\times 400$} & 4 & 1800 & 7.6 & 590 & 2.0 & 590 & 2.0 \\
\hline & 8 & 4075 & 29.9 & 733 & 4.0 & 733 & 4.0 \\
\hline & 16 & 10547 & 82.4 & 1170 & 6.0 & 1170 & 6.0 \\
\hline & 24 & 17303 & 163.3 & 1282 & 8.0 & 1282 & 8.0 \\
\hline \multirow[t]{4}{*}{$\operatorname{rot} 300 \times 400$} & 4 & 645 & 6.0 & 238 & 2.0 & 238 & 2.0 \\
\hline & 8 & 1945 & 26.6 & 357 & 4.0 & 357 & 4.0 \\
\hline & 16 & 6073 & 67.1 & 784 & 5.5 & 784 & 5.5 \\
\hline & 24 & 9756 & 124.2 & 819 & 7.5 & 819 & 7.5 \\
\hline \multirow[t]{4}{*}{$\operatorname{irt} 300 \times 400$} & 4 & 27430 & 8.0 & 10112 & 2.0 & 10112 & 2.0 \\
\hline & 8 & 79212 & 31.2 & 11963 & 4.0 & 11963 & 4.0 \\
\hline & 16 & 145351 & 94.0 & 11813 & 6.0 & 11813 & 6.0 \\
\hline & 24 & 175723 & 191.8 & 9198 & 8.0 & 9198 & 8.0 \\
\hline \multicolumn{8}{|l|}{$2 D-A 2 A$} \\
\hline \multirow[t]{4}{*}{ iso300 $\times 400$} & 4 & 1405 & 8.0 & 557 & 2.0 & 557 & 2.0 \\
\hline & 8 & 6225 & 24.0 & 1034 & 3.0 & 1034 & 3.0 \\
\hline & 16 & 20701 & 64.0 & 1867 & 4.0 & 1867 & 4.0 \\
\hline & 24 & 37169 & 120.0 & 2413 & 5.0 & 2413 & 5.0 \\
\hline \multirow[t]{4}{*}{$\operatorname{rot} 300 \times 400$} & 4 & 655 & 8.0 & 246 & 2.0 & 246 & 2.0 \\
\hline & 8 & 2692 & 24.0 & 480 & 3.0 & 480 & 3.0 \\
\hline & 16 & 10524 & 64.0 & 989 & 4.0 & 989 & 4.0 \\
\hline & 24 & 19555 & 120.0 & 1239 & 5.0 & 1239 & 5.0 \\
\hline \multirow[t]{4}{*}{$\operatorname{irt} 300 \times 400$} & 4 & 27346 & 8.0 & 10107 & 2.0 & 10107 & 2.0 \\
\hline & 8 & 121924 & 24.0 & 18252 & 3.0 & 18252 & 3.0 \\
\hline & 16 & 259999 & 64.0 & 18478 & 4.0 & 18478 & 4.0 \\
\hline & 24 & 391431 & 120.0 & 18341 & 5.0 & 18341 & 5.0 \\
\hline
\end{tabular}

performance and convergence results obtained from these runs are displayed in the following tables and bar charts. In all tables and figures, "P2P" and "A2A" refer to the pointto-point and all-to-all communication schemes, respectively. In $2 \mathrm{D}$ partitionings, the number of processors in rows and columns of the processor mesh are not restricted to be powers of two. Therefore, all-to-all communication primitives needed in fold and expand operations are implemented using the all-to-all broadcast algorithm proposed by Jacunski et al. [16].

\subsection{Per iteration performance}

Table 2 displays per iteration run times of the parallel implementations of BSCM and PSCM, and the serial run time of BSCM. The bottom of the table displays the speedup values averaged over all instances for each possible number of processors. The per iteration run time of BSCM is taken as the sequential run time in calculating the speedups. The bar charts for the individual speedup values are displayed in Fig. 8 for $300 \times 400$ images. 
Table 4

Communication patterns of the 2D partitionings_-dissected into fold and expand phases-for the $H$ matrices given in Table 3

\begin{tabular}{|c|c|c|c|c|c|c|c|c|c|}
\hline \multirow[t]{3}{*}{$H$ matrix } & \multirow[t]{3}{*}{$K$} & \multicolumn{4}{|c|}{ Expand $H x /$ Fold $H^{T} \pi$} & \multicolumn{4}{|c|}{ Fold $H x$ / Expand $H^{T} \pi$} \\
\hline & & \multicolumn{2}{|l|}{ Volume } & \multicolumn{2}{|c|}{ Number } & \multicolumn{2}{|l|}{ Volume } & \multicolumn{2}{|c|}{ Number } \\
\hline & & Total & Max & Total & Max & Total & Max & Total & Max \\
\hline \multicolumn{10}{|l|}{$P 2 P$} \\
\hline \multirow[t]{4}{*}{ iso300 $\times 400$} & 4 & 678 & 237 & 3.6 & 1.0 & 1122 & 354 & 4.0 & 1.0 \\
\hline & 8 & 2223 & 452 & 21.9 & 3.0 & 1852 & 346 & 8.0 & 1.0 \\
\hline & 16 & 1878 & 276 & 33.6 & 2.9 & 8717 & 1095 & 48.0 & 3.0 \\
\hline & 24 & 3700 & 322 & 91.3 & 5.0 & 13603 & 1074 & 72.0 & 3.0 \\
\hline \multirow[t]{4}{*}{$\operatorname{rot} 300 \times 400$} & 4 & 197 & 99 & 2.0 & 1.0 & 448 & 140 & 4.0 & 1.0 \\
\hline & 8 & 829 & 153 & 18.6 & 3.0 & 1116 & 215 & 8.0 & 1.0 \\
\hline & 16 & 818 & 148 & 19.1 & 2.5 & 5255 & 712 & 48.0 & 3.0 \\
\hline & 24 & 1458 & 133 & 52.2 & 4.5 & 8298 & 746 & 72.0 & 3.0 \\
\hline \multirow[t]{4}{*}{$\operatorname{irt} 300 \times 400$} & 4 & 12157 & 5920 & 4.0 & 1.0 & 15273 & 4192 & 4.0 & 1.0 \\
\hline & 8 & 44535 & 7744 & 23.2 & 3.0 & 34677 & 5597 & 8.0 & 1.0 \\
\hline & 16 & 44722 & 4237 & 46.0 & 3.0 & 100629 & 8984 & 48.0 & 3.0 \\
\hline & 24 & 77991 & 4034 & 119.8 & 5.0 & 97733 & 5313 & 72.0 & 3.0 \\
\hline \multicolumn{10}{|l|}{$A 2 A$} \\
\hline \multirow[t]{4}{*}{ iso $300 \times 400$} & 4 & 597 & 296 & 4.0 & 1.0 & 808 & 260 & 4.0 & 1.0 \\
\hline & 8 & 4410 & 637 & 16.0 & 2.0 & 1815 & 396 & 8.0 & 1.0 \\
\hline & 16 & 4313 & 493 & 32.0 & 2.0 & 16388 & 1373 & 32.0 & 2.0 \\
\hline & 24 & 10924 & 833 & 72.0 & 3.0 & 26246 & 1581 & 48.0 & 2.0 \\
\hline \multirow[t]{4}{*}{$\operatorname{rot} 300 \times 400$} & 4 & 201 & 100 & 4.0 & 1.0 & 454 & 146 & 4.0 & 1.0 \\
\hline & 8 & 1625 & 278 & 16.0 & 2.0 & 1067 & 203 & 8.0 & 1.0 \\
\hline & 16 & 1632 & 221 & 32.0 & 2.0 & 8892 & 768 & 32.0 & 2.0 \\
\hline & 24 & 4280 & 312 & 72.0 & 3.0 & 15275 & 928 & 48.0 & 2.0 \\
\hline \multirow[t]{4}{*}{$\operatorname{irt} 300 \times 400$} & 4 & 12144 & 5921 & 4.0 & 1.0 & 15202 & 4186 & 4.0 & 1.0 \\
\hline & 8 & 87538 & 12874 & 16.0 & 2.0 & 34386 & 5378 & 8.0 & 1.0 \\
\hline & 16 & 87635 & 6858 & 32.0 & 2.0 & 172364 & 11620 & 32.0 & 2.0 \\
\hline & 24 & 226086 & 10275 & 72.0 & 3.0 & 165345 & 8066 & 48.0 & 2.0 \\
\hline
\end{tabular}

Processors are organized into dimensional meshes of size $2 \times 2$ for $K=4,4 \times 2$ for $K=8,4 \times 4$ for $K=16$, and $6 \times 4$ for $K=24$.

As seen in Table 2, the 2D-P2P scheme leads to better performance than the other two schemes. In particular, the 2D$\mathrm{P} 2 \mathrm{P}$ scheme leads to faster execution in 34 and 31 instances out of 36 instances for parallel BSCM and parallel PSCM, respectively. The 1D-P2P scheme obtains faster execution times in only 5 instances for both BSCM and PSCM. The 2D-A2A scheme is the fastest only in 4-way parallelization of PSCM for iso600 $\times 800$ and irt600 $\times 800$. As seen in Table 2 , BSCM and PSCM display comparable parallel performance, where BSCM performs slightly better on the average. This performance difference slightly increases in favor of parallel BSCM in 2D partitioning. These experimental findings were expected because PSCM incurs extra computation as discussed in Section 3. Moreover, parallel PSCM requires an extra communication along the rows of processor mesh for computing the $\mu^{k}$ and $\gamma^{k}$ values in 2D partitioning as seen in Fig. 6 .

As seen in Table 2 and Fig. 8, among the blur types used, the data sets produced by the isotropic and rotation blur lead to comparable speedup values, whereas the data sets produced by combined blur lead to inferior parallel performance. This phenomenon can be attributed to the absence of columns with only one nonzero in "irt" matrices, i.e., these matrices are likely to yield harder partitioning instances in terms of communication overhead minimization.

Tables 3 and 4 are presented in order to further investigate the effect of the matrix partitioning schemes on the parallel performance of BSCM and PSCM. Table 3 displays the communication patterns in the parallel matrix-vector and matrixtranspose-vector multiplies obtained by the $1 \mathrm{D}$ and $2 \mathrm{D}$ partitioning schemes for the images of size $300 \times 400$. Table 4 shows the dissection of the communication patterns into expand and fold phases for $2 \mathrm{D}$ schemes.

In Tables 3 and 4, message-volume values are given in terms of the words communicated. In terms of the total communication volume, the $1 \mathrm{D}$ partitioning scheme produces the best partitions in 7 out of 12 instances, whereas the 2D-P2P scheme produces the best partitions in 8-, 16-, and 24-way partitionings of irt300 $\times 400$, and the $2 \mathrm{D}-\mathrm{A} 2 \mathrm{~A}$ scheme produces best partitions in 4-way partitioning of iso300 $\times 400$ and irt300 $\times 400$. This experimental outcome may be due to the fact that $1 \mathrm{D}$ rowwise partitioning disturbs only column coherence, whereas the 2D partitioning schemes disturb both row and column coherences. In terms of the total number of messages, 1D scheme competes with the 2D-A2A scheme, where 2D-P2P displays 
Table 5

The number of iterations to convergence for BSCM and parallel PSCM

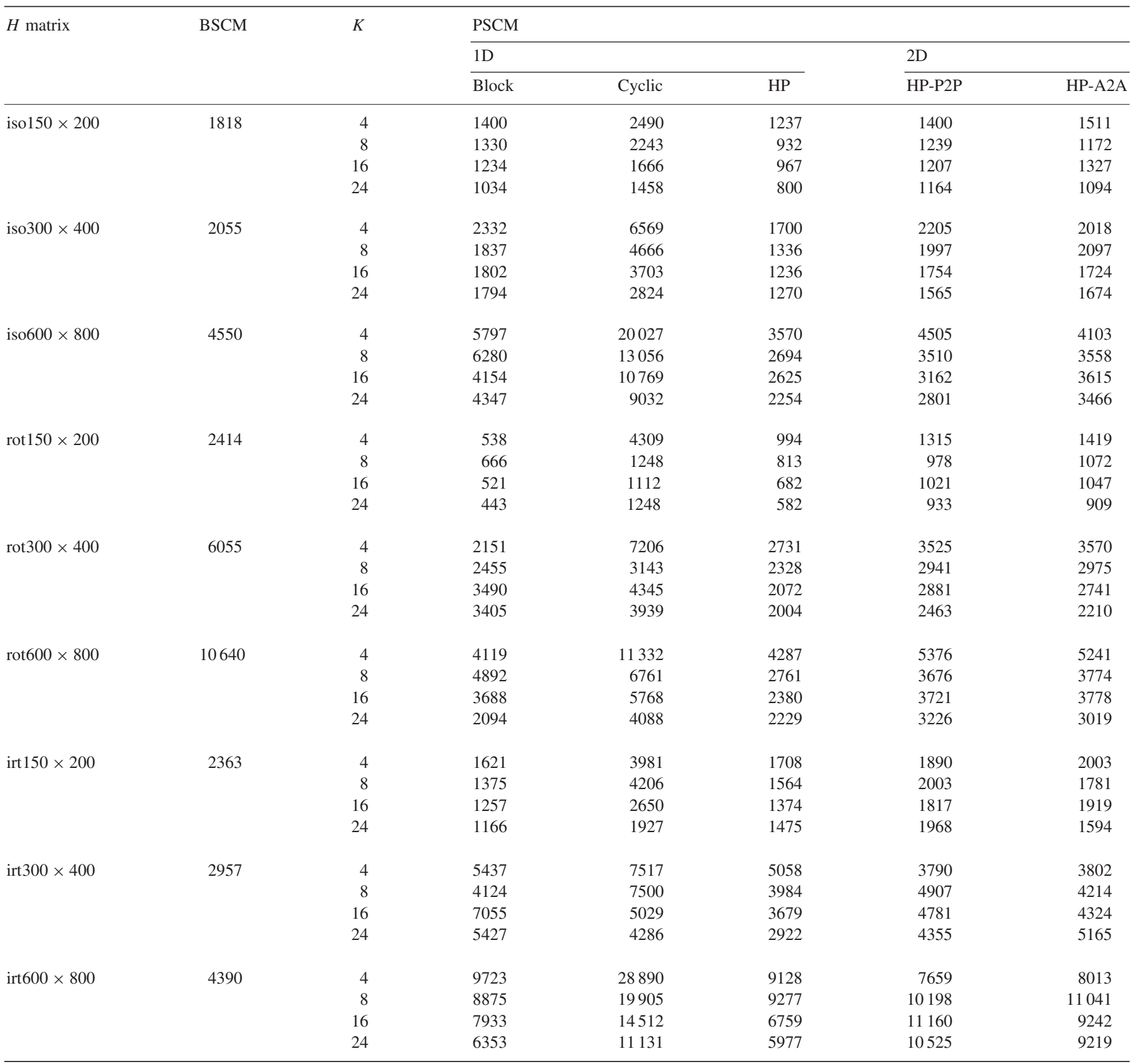

the worst performance. The 1D and 2D-A2A schemes produce, respectively, the best partitions in 9 and 3 instances out of 12 instances. The performance of the 1D partitioning scheme relies on the enhancement given in [34]. The relatively better performance of $2 \mathrm{D}-\mathrm{A} 2 \mathrm{~A}$ is expected as discussed in Section 5.3. Note that the number of messages in $2 \mathrm{D}-\mathrm{A} 2 \mathrm{~A}$ is always the same for a given number of processors because of the regular communication operations. In terms of maximum message volume, the 1D scheme produces best results in all partitioning instances of $\operatorname{rot} 300 \times 400$ and 8-, 16-, and 24 -way partitioning of iso300 $\times 400$, whereas $2 \mathrm{D}$-P2P pro- duces best results in all partitioning instances of irt300 $\times 400$ and 4-way partitioning of iso $300 \times 400$. This relatively better performance of the 1D scheme can also be attributed to the enhancement [34] which involves explicit effort towards balancing the communication-volume loads of processors. In terms of maximum number of messages handled by a single processor, the $2 \mathrm{D}$ schemes produce considerably better results than the $1 \mathrm{D}$ scheme, where $2 \mathrm{D}-\mathrm{A} 2 \mathrm{~A}$ is slightly better than 2D-P2P.

Combining these experimental outcomes for communication pattern results and considering the interconnection 

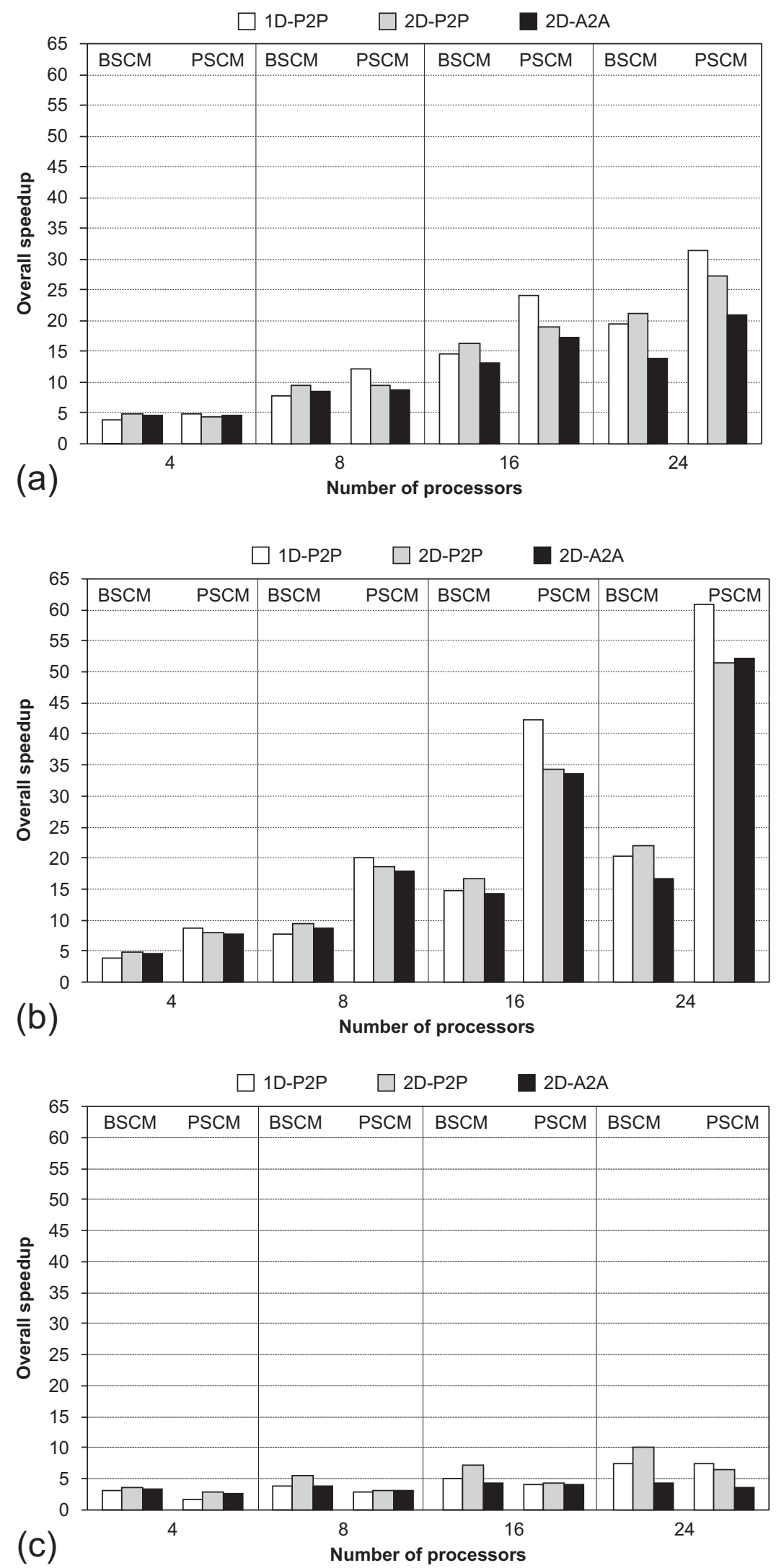

Fig. 9. Overall speedup charts of BSCM and PSCM for the images of size $300 \times 400$ : (a) isotropic blur; (b) rotation blur; (c) combined blur. 
Table 6

Preprocessing times for the images of size $400 \times 300$, expressed in terms of the per iteration times of the respective PSCM implementation

\begin{tabular}{|c|c|c|c|c|}
\hline \multirow[t]{2}{*}{$H$ matrix } & \multirow[t]{2}{*}{$K$} & \multicolumn{3}{|c|}{ Partitioning scheme } \\
\hline & & 1D-P2P & 2D-P2P & 2D-A2A \\
\hline \multirow[t]{4}{*}{ iso $400 \times 300$} & 4 & 125 & 115 & 220 \\
\hline & 8 & 291 & 302 & 149 \\
\hline & 16 & 618 & 835 & 110 \\
\hline & 24 & 912 & 1287 & 81 \\
\hline \multirow[t]{4}{*}{$\operatorname{rot} 400 \times 300$} & 4 & 68 & 83 & 144 \\
\hline & 8 & 184 & 248 & 110 \\
\hline & 16 & 458 & 553 & 70 \\
\hline & 24 & 699 & 858 & 55 \\
\hline \multirow[t]{4}{*}{$\operatorname{irt} 400 \times 300$} & 4 & 335 & 230 & 514 \\
\hline & 8 & 1413 & 596 & 494 \\
\hline & 16 & 4093 & 1290 & 258 \\
\hline & 24 & 5228 & 3106 & 247 \\
\hline
\end{tabular}

network of our PC cluster, we expect 2D-P2P to be the best because of its lower number of message requirements and moderate communication volume requirements. In fact, the parallel performance of BSCM and PSCM given in Table 2 and Fig. 8 confirm those expectations. However, depending on the machine architecture, the aforementioned communication metrics would have different impacts on the parallel performance of BSCM and PSCM. For example, on an interconnection network with a high communication bandwidth, 2D-A2A may perform better than 2D-P2P since it mainly suffers from high communication volume.

\subsection{Overall performance}

In our experiments, the tolerance parameter was set to $0.8 \%$ of the mean value of the observed image. In general, the smaller the tolerance parameter $\varepsilon$, the better the quality of the restored images, and the larger the iteration numbers. For 0-255 grayscale images, this value of the tolerance parameter provides high quality restorations. The relaxation parameter $\lambda$ was taken as 1.7 as in [35]. The algorithms were initialized with the zero vector meaning that every pixel in the image to be recovered was assumed to be initially black. With these values, the number of iterations required for convergence are given in Table 5 for the proposed partitioning schemes. The overall convergence results for partitionings based on natural ordering are also included. The reason for this inclusion is to demonstrate that the partitioning schemes used for achieving efficient parallel implementations do not degrade overall convergence performance. In Table 5, "Block" and "Cyclic" refer to block-striped and cyclic partitionings applied to the natural row order of the $H$ matrix. Finally, "HP" denotes the partitionings obtained using hypergraph models.

Comparison of PSCM and BSCM highlights the fact that increasing number of blocks reduces the number of iterations to convergence, in general. Furthermore, comparison of PSCM results for 1D partitionings shows that HP-based partitionings do not have a negative impact on the number of iterations to convergence. Comparison of $1 \mathrm{D}$ and $2 \mathrm{D}$ results reveals that 2D leads to larger number of iterations for a given number of processors. This is to be expected since the number of row blocks determines the speed of convergence of PSCM. Recall that the number of row blocks in a 2D mesh of $K=$ $R \times C$ processors is $R$ as opposed to $K$ in a $1 \mathrm{D} K$-way partitioning.

Fig. 9 displays the overall speedup values of parallel BSCM and PSCM for the images of size $300 \times 400$. As seen in the figure, $\mathrm{PSCM}$ is superior to BSCM in all instances except for the irt300 $\times 400$ matrix. Although 2D-P2P leads to better speedup on the per iteration basis, the winner with respect to overall performance is not clear. Note that the superlinear speedups for the isotropic and rotation cases are because of the reduced number of iterations.

Finally, we consider the preprocessing overhead of our parallel implementations. Table 6 gives the partitioning times of the matrices expressed in terms of the per iteration run time of the PSCM. This table shows that the cost of preprocessing is amortized in achieving accurate results.

\subsection{Restoration results}

We evaluate the restoration performance of the parallel methods using the three images shown in Fig. 10(a). Blurred image $g$ is generated using Eq. (2), or by simply multiplying $f$ (original image) with $H$ so that $g=H f$ and adding the noise described earlier in this section. In Fig. 10(b)-(d), the resulting distorted images are shown for the isotropic, rotational, and combined blurs, respectively.

With the same parameter values given earlier, we have restored the images by the surrogate constraint methods. The results corresponding to the isotropic, rotational, and combined blurs are given in Fig. 10(e)-(g). 


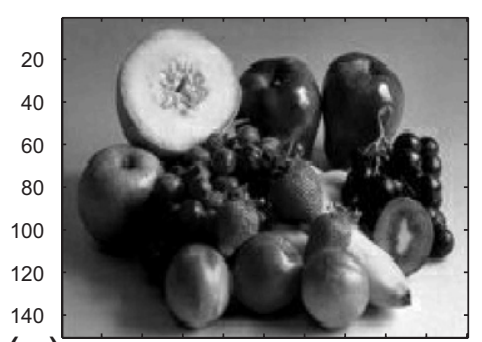

(a) 20406080100120140160180200

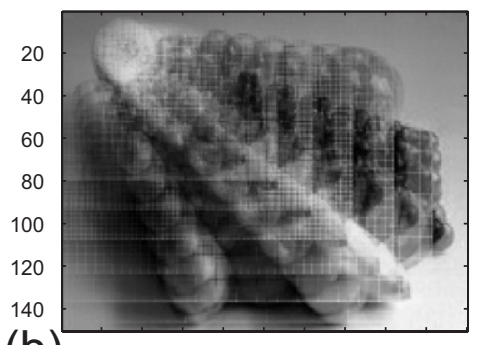

(b) $2040 \quad 6080100120140160180200$

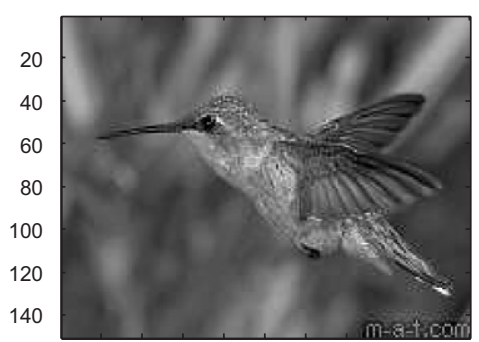

20406080100120140160180200

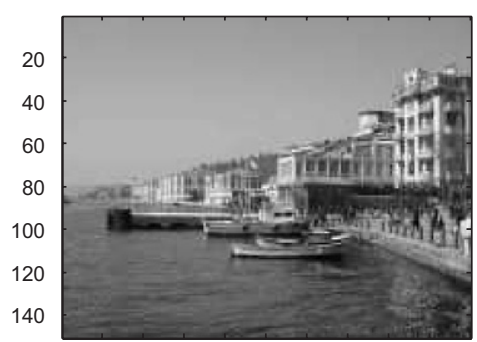

20406080100120140160180200
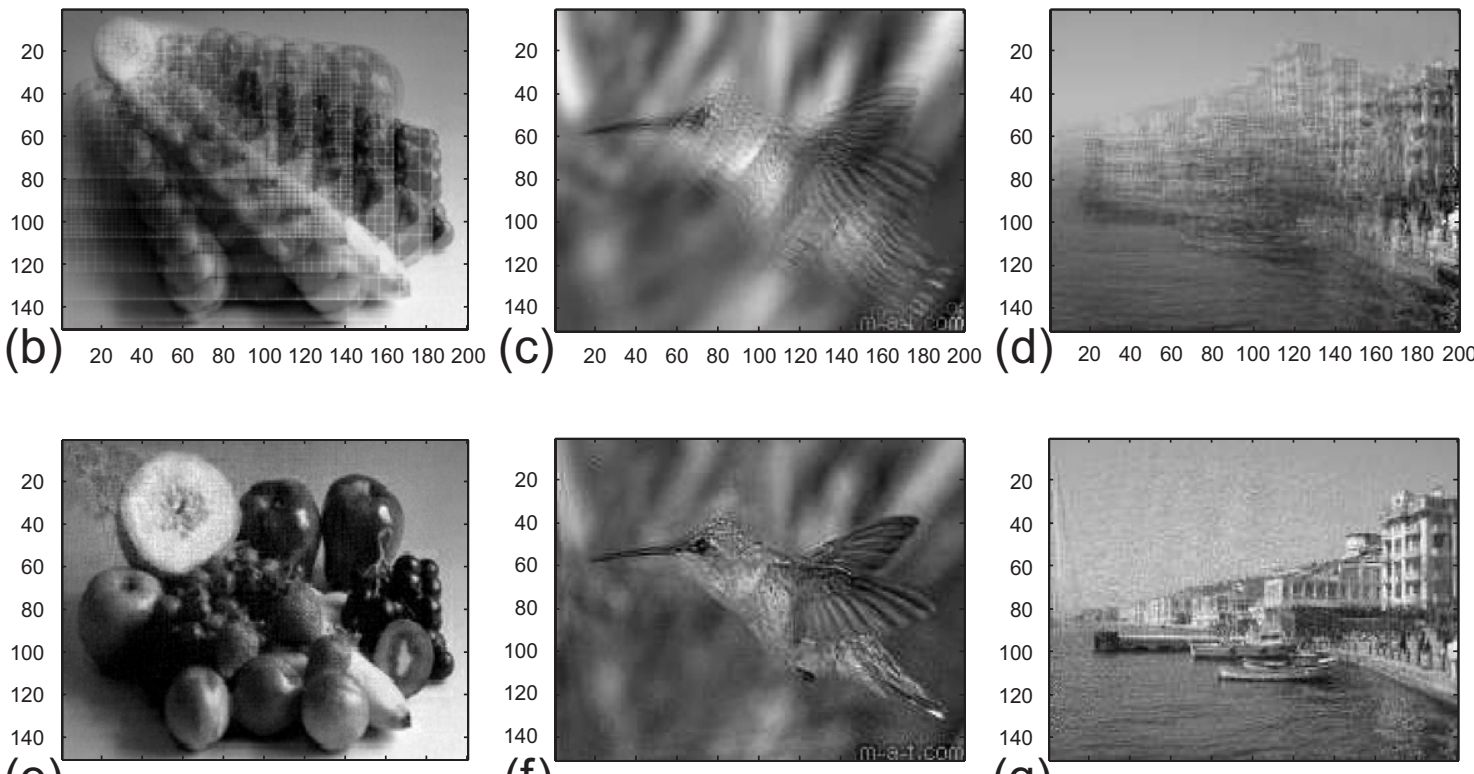

(e) $2040 \quad 6080100120140160180200$
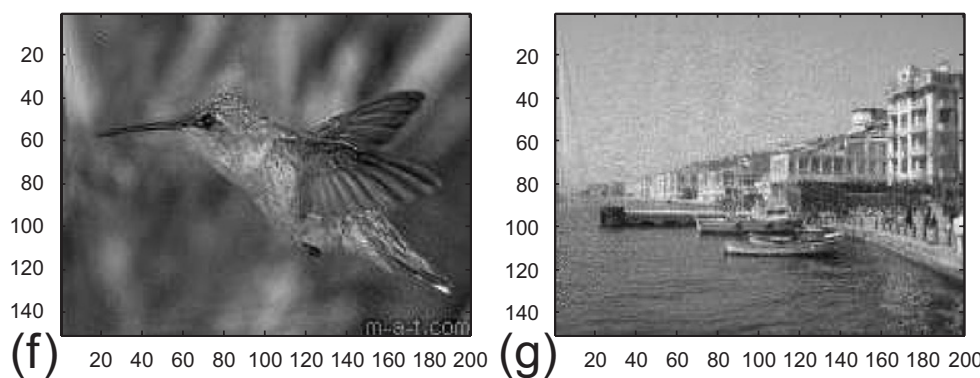

Fig. 10. Blurred and restored images: (a) original images; (b) noisy isotropic blur; (c) noisy rotational blur; (d) noisy combined blur; (e) restore b; (f) restore $\mathrm{c} ;(\mathrm{g})$ restore $\mathrm{d}$.

\section{Conclusion}

We studied the image restoration problem by formulating it as a system of linear inequalities. We used the surrogate constraint methods which are well suited to large problems and amenable to parallelization. The study concentrated on BSCM, which is the basic method, and on an improved version of the parallel method PSCM. We developed several parallel implementations. For efficient parallelization based on 1D and 2D partitionings of the coefficient matrix, we used state-of-the-art hypergraph partitioning schemes that minimize communication overhead while maintaining the load balance. Restoration abilities of the surrogate constraint implementations are validated using the parallel implementations for restoring severely blurred images.

The parallel implementation of BSCM was observed to produce better results compared with PSCM as far as the per iteration performance is concerned. However, increasing the number of blocks accelerates the speed of convergence significantly, hence PSCM outperforms BSCM with respect to the overall performance. In parallel PSCM, although 2D partitioning scheme leads to better speedup than the 1D scheme on the per iteration basis, 1D partitioning scheme performs comparably based on overall performance.

Note that satisfactory restorations can be achieved by decreasing the tolerance parameter at the expense of increased running time. Actually, the system parameters can be set according to the requirements of the application. Moreover, the iterative restoration technique has the advantage that the image can be viewed during the restoration process, and the process can be terminated as soon as the restoration level satisfies the application requirements.

\section{Acknowledgment}

We are indebted to anonymous referees whose comments helped improve the presentation of Section 6 .

\section{References}

[1] G. Burns, R. Daoud, J. Vaigl, LAM: an open cluster environment for MPI, in: J.W. Ross (Ed.), Proceedings of Supercomputing Symposium, 1994, pp. 179-186. 
[2] Ü.V. Çatalyürek, Hypergraph models for sparse matrix partitioning and reordering, s.D. Thesis, Computer Engineering and Information Sciences, Bilkent University, 1999.

[3] Ü.V. Çatalyürek, C. Aykanat, Decomposing irregularly sparse matrices for parallel matrix-vector multiplication, Lecture Notes in Computer Science, vol. 1117, 1996, pp. 75-86.

[4] Ü.V. Çatalyürek, C. Aykanat, Hypergraph-partitioning-based decomposition for parallel sparse-matrix vector multiplication, IEEE Trans. Parallel and Distributed Systems 10 (1999) 673-693.

[5] Ü.V. Çatalyürek, C. Aykanat, PaToH: a multilevel hypergraph partitioning tool, version 3.0, Technical Report BU-CE-9915, Computer Engineering Department, Bilkent University, 1999.

[6] Ü.V. Çatalyürek, C. Aykanat, A hypergraph-partitioning approach for coarse-grain decomposition, in: Proceedings of Scientific Computing 2001 (SC2001), Denver, Colorado, 2001, pp. 10-16.

[7] Y. Censor, T. Elfving, New method for linear inequalities, Linear Algebra Appl. 42 (1982) 199-211.

[8] Y. Censor, S.A. Zenios, Parallel Optimization: Theory, Algorithms, and Applications, Oxford University Press, Oxford, 1997.

[9] U.M. García-Palomares, F.J. Gonzalez-Castaño, Acceleration technique for solving convex (linear) systems via projection methods, Technical Report, Escola Tecnica Superior de Enxeneiros de Telecomunicacion, 1996.

[10] M.R. Garey, D.S. Johnson, Computers and Intractability: A Guide to the Theory of NP-Completeness, W. H. Freeman \& Co., New York, NY, USA, 1979.

[11] L.G. Gubin, B.T. Polyak, E.V. Raik, The method of projections for finding the common point of convex sets, USSR Comput. Math. and Math. Phys. 6 (1967) 326-333.

[12] M. Hanke, Conjugate gradient type methods for ill-posed problems, Pitman Research Notes in Mathematics Series, Longman Scientific and Technical, Essex CM20 2JE, England, 1995.

[13] B. Hendrickson, T.G. Kolda, Partitioning rectangular and structurally nonsymmetric sparse matrices for parallel processing, SIAM J. Sci. Comput. 21 (1998) 2048-2072.

[14] B. Hendrickson, T.G. Kolda, Graph partitioning models for parallel computing, Parallel Comput. 26 (2000) 1519-1534.

[15] B. Hendrickson, R. Leland, S. Plimpton, An efficient parallel algorithm for matrix-vector multiplication, Internat. J. High Speed Comput. 7 (1995) 73-88.

[16] M. Jacunski, P. Sadayappan, D. K. Panda, All-to-all broadcast on switchbased clusters of workstations, in: IPPS '99/SPDP '99, Proceedings of the 13th International Symposium on Parallel Processing and the 10th Symposium on Parallel and Distributed Processing, IEEE Computer Society, Washington, DC, USA, 1999, pp. 325-329.

[17] G. Karypis, V. Kumar, MeTiS: a software package for Partitioning Unstructured Graphs, Partitioning Meshes, and Computing FillReducing Orderings of Sparse Matrices Version 4.0, University of Minnesota, Department of Computer Science/Army HPC Research Center, Minneapolis, September 1998.

[18] G. Karypis, V. Kumar, Multilevel algorithms for multi-constraint graph partitioning, Technical Report 98-019, University of Minnesota, Department of Computer Science/Army HPC Research Center, Minneapolis, May 1998.

[19] G. Karypis, V. Kumar, Multilevel algorithms for multi-constraint hypergraph partitioning, Technical Report 99-034, University of Minnesota, Department of Computer Science/Army HPC Research Center, Minneapolis, November 1998.

[20] V. Kumar, A. Grama, A. Gupta, G. Karypis, Introduction to Parallel Computing: Design and Analysis of Algorithms, The Benjamin/Cummings, Menlo Park, CA, 1994.

[21] R.L. Lagendijk, J. Biemond, Iterative Identification and Restoration of Images, Kluwer Academic Publishers, Dordrecht, MA, 1991.

[22] K.P. Lee, J.G. Nagy, Steepest descent, CG and iterative regularization of ill-posed problems, BIT 43 (2003) 1003-1017.

[23] T. Lengauer, Combinatorial Algorithms for Integrated Circuit Layout, Wiley-Teubner, Chichester, UK, 1990.

[24] J.G. Lewis, D.G. Payne, R.A. van de Geijn, Matrix-vector multiplication and conjugate gradient algorithms on distributed memory computers, in:
Proceedings of the Scalable High Performance Computing Conference, Knoxville, TN, USA, 1994, pp. 542-550.

[25] J.G. Lewis, R.A. van de Geijn, Distributed memory matrix-vector multiplication and conjugate gradient algorithms, in: Proceedings of the 1993 ACM/IEEE conference on Supercomputing, IEEE, Portland, Oregon, USA, 1993, pp. 484-492.

[26] J.G. Nagy, D.P. O'Leary, Fast iterative image restoration with a spatially-varying PSF, in: F.T. Luk (Ed.), Advanced Signal Processing Algorithms, Architectures, and Implementations IV, vol. 3162, 1997, pp. 388-399.

[27] J.G. Nagy, D.P. O'Leary, Restoring images degraded by spatially-variant blur, SIAM J. Sci. Comput. 19 (1998) 1063-1082.

[28] J.G. Nagy, K. Palmer, L. Perrone, Iterative methods for image deblurring: a Matlab object oriented approach, Numer. Algorithms 36 (2004) 73-93.

[29] H. Özaktaş, Algorithms for linear and convex feasibility problems: a brief study of iterative projection, localization and subgradient methods, $\mathrm{Ph} . \mathrm{D}$. Thesis, Department of Industrial Engineering, Bilkent University, 1998.

[30] H. Özaktaş, M.Ç. Pınar, M. Akgül, The parallel surrogate constraint approach to the linear feasibility problem, Lecture Notes in Comput. Sci. 1184 (1996) 565-574.

[31] H. Özaktaş, M.Ç. Pınar, M. Akgül, Restoration of space-variant global blurs caused by severe camera movements and coordinate distortions, J. Optics 29 (1998) 303-310.

[32] Y. Saad, Iterative Methods for Sparse Linear Systems, PWS Publishing Co., Boston, 1996.

[33] R.S. Tuminaro, J.N. Shadid, S.A. Hutchinson, Parallel sparse matrix vector multiply software for matrices with data locality, Concurrency: Practice and Experience 10 (1998) 229-247.

[34] B. Uçar, C. Aykanat, Encapsulating multiple communication-cost metrics in partitioning sparse rectangular matrices for parallel matrix-vector multiplies, SIAM J. Sci. Comput. 25 (2004) 1827-1859.

[35] K. Yang, K.G. Murty, New iterative methods for linear inequalities, J. Optim. Theory Appl. 72 (1992) 163-185.

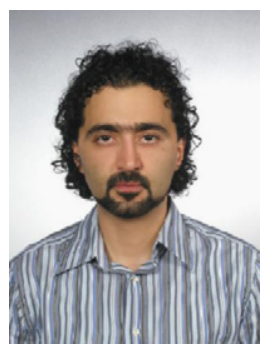

Bora Ucar received the Ph.D. degree (2005) in Computer Engineering from Bilkent University, Ankara, Turkey. His research interests are combinatorial scientific computing and high performance computing.

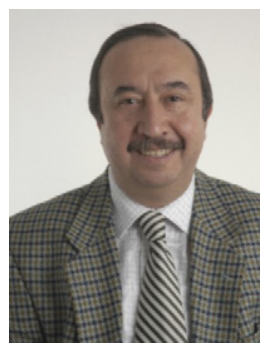

Cevdet Aykanat received the B.S. and M.S. degrees from Middle East Technical University, Ankara, Turkey, both in electrical engineering, and the Ph.D. degree from Ohio State University, Columbus, in electrical and computer engineering. He was a Fulbright scholar during his $\mathrm{Ph} . \mathrm{D}$. studies. He worked at the Intel Supercomputer Systems Division, Beaverton, Oregon, as a research associate. Since 1989 , he has been affiliated with the Department of Computer Engineering, Bilkent University, Ankara, Turkey, where he is currently a professor. His research interests mainly include parallel computing, parallel scientific computing and its combinatorial aspects, parallel computer graphics applications, parallel data mining, graph and hypergraph-partitioning, load balancing, neural network algorithms, high performance information retrieval systems, parallel and distributed web crawling, parallel and distributed databases, and grid computing. He has (co)authored over 40 technical papers published in academic journals indexed in SCI. He is the recipient of the 1995 Young Investigator Award of The Scientific and Technical Research Council of Turkey. He is a member of the ACM and the IEEE Computer Society. He has been recently appointed as a member of IFIP Working Group 10.3 (Concurrent Systems) and INTAS Council of Scientists. 


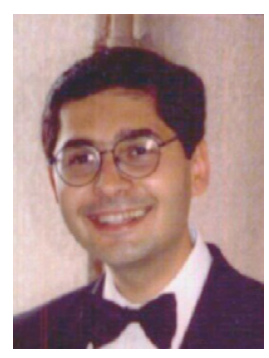

Mustafa Ç. Pinar received the Ph.D. degree (1992) in Systems Engineering from the University of Pennsylvania. His research interests are Applied Optimization and Scientific Computing. $\mathrm{He}$ is a professor in the Industrial Engineering Department of Bilkent University, Ankara, Turkey.

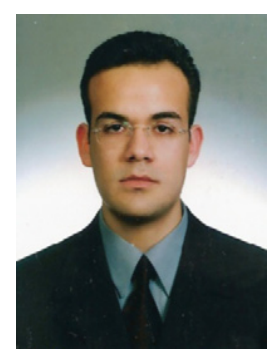

Tahir Malas received his M.Sc. degree in 2004 from Computer Engineering Department of Bilkent University, Ankara, Turkey. Currently he is working towards the Ph.D. degree in the Department of Electrical and Electronics Engineering of Bilkent University. His current working area is in computational electromagnetics; in particular he deals with preconditioning methods for fast solvers. 